\title{
Machine learning model and strategy for fast and accurate detection of leaks in water supply network
}

\author{
Xudong Fan ${ }^{1}$, Xijin Zhang ${ }^{2}$ and Xiong ( Bill) $\mathrm{Yu}^{3^{*}}$ [D
}

\begin{abstract}
The water supply network (WSN) is subjected to leaks that compromise its service to the communities, which, however, is challenging to identify with conventional approaches before the consequences surface. This study developed Machine Learning (ML) models to detect leaks in the WDN. Water pressure data under leaking versus non-leaking conditions were generated with holistic WSN simulation code EPANET considering factors such as the fluctuating user demands, data noise, and the extent of leaks, etc. The results indicate that Artificial Neural Network (ANN), a supervised ML model, can accurately classify leaking versus non-leaking conditions; it, however, requires balanced dataset under both leaking and non-leaking conditions, which is difficult for a real WSN that mostly operate under normal service condition. Autoencoder neural network (AE), an unsupervised ML model, is further developed to detect leak with unbalanced data. The results show AE ML model achieved high accuracy when leaks occur in pipes inside the sensor monitoring area, while the accuracy is compromised otherwise. This observation will provide guidelines to deploy monitoring sensors to cover the desired monitoring area. A novel strategy is proposed based on multiple independent detection attempts to further increase the reliability of leak detection by the AE and is found to significantly reduce the probability of false alarm. The trained AE model and leak detection strategy is further tested on a testbed WSN and achieved promising results. The ML model and leak detection strategy can be readily deployed for in-service WSNs using data obtained with internet-of-things (loTs) technologies such as smart meters.
\end{abstract}

Keywords: Water supply network, Artificial intelligence, Machine learning, Artificial neural network, Autoencoder neural network, Leak detection

\section{Introduction}

Water supply system provides one of the most essential service for the communities. However, due to the deterioration of the underground water pipe network, a large amount of water is lost every year, mostly unnoticed. According to [38], about 3281 megaliters $\left(10^{6}\right)$ of water was wasted in the UK during 2009-2011, and about 15\% of supplied drinking water was wasted each year in the US. The percentage of lost water is significantly higher

\footnotetext{
* Correspondence: xxy21@case.edu

${ }^{3}$ Department of Civil and Environmental Engineering, Case Western Reserve University, 2104 Adelbert Road, Bingham 206, Cleveland, OH 44106-7201, USA

Full list of author information is available at the end of the article
}

in historical water districts such as Cleveland, Ohio, Boston, MA, etc. Many factors can cause leaks, such as pipe corrosion, aging, defects and inappropriate installation [21]. A detailed discussion is presented about the cause of water main failures by Sadiq et.al [39]. Due to the complex underground environment, predicting underground water pipe failure remains a challenging problem. The state of practice with most agencies is to rehabilitate pipes after leaks are directly observed [47], while many small leaks remain undetected until the damages surfaced in the form of ground cavitation etc. Evidently, the agency perspective on cost does not include the socio-economic cost to the communities such 
as healthcare cost due to compromised water quality. Technologies to detect leakage and forecast water pipe failure will enable agencies to evolve into preventative strategies with significant socio-economic benefits.

A significant amount of efforts has been made on water pipe leak detection. Strategies can be broadly classified in five categories, i.e., vision based, sensor/instrumental based, transient response based, model based, and data-based [10]. The first two technologies require to use specialized mobile inspection equipment with optical, acoustic, or electromagnetic sensors [16, 20,34 ], which is expensive and time-consuming. For example, leak detection with acoustic signals can often be influenced by the type of leak, opening size, pipe materials and soil conditions [8]. Technology such as ground penetrating radar can detect leak around pipe but requires heavy human involvement in signal analyses $[3,5,14]$. The pressure or acoustic transient signals are used for pipe burst detection, since such transient signals travel along the pipe at the speed of sound starting from the burst location [41]. However, the transient responses decay with distance and diminish over a short time, and therefore requires sensor with high spatial and temporal resolution. Model-based approach for leak detection has been theoretically shown to be capable of identifying leakage and localize its position. They are, however, very difficult to be implemented in real systems $[2,12,28]$ due to its requirements on detailed information required for a hydraulic model such as the user demand, pipe condition, water pressure distribution, etc. Such information is difficult to collect or is typically not available. Empowered with the Internet of Things (IoT) and artificial intelligence (AI), data-driven technologies have been proven capability knowledge discovery [24], image processing [7], and event forecasting, etc. [25]. Data-driven leak detection, which is based on learning from historical data with statistical or pattern recognition algorithms, is emerging [37]. It does not require collecting comprehensive set of information as needed for a model based approach.

With the development of supervisory control and data acquisition (SCADA) systems, real-time monitoring data of water pressure and/or flow rate are available and can be collected for the leak detection and localization [11, $22,43]$. Other data such as monitored acoustic sensor data was found significantly affected by the environmental noise and limited transmission distance [27, 42]. The monitored water pressure data of districted metering areas (DMA) can be trained with a state of art ML model to detect possible leak by used of the historical data, which is combined with traditional methods such as vision-based or instrument-based inspection to pinpoint leak location. Different ML algorithms have been developed for leaks detection, for example the ANNs and a fully-line DensNet [30-32, 50]. The leaks or bursts were also detected through comparing the predicted water demand or the water pressure at nodes versus the actual demand or pressure $[4,49]$.

The existing ML algorithms for leak detection generally include classification model, prediction-classification model, and statistical model [48], each featuring advantages and limitations. The classification model uses supervised learning model and requires large datasets under both normal conditions and leaking conditions. In the practice, data under leaking conditions is generally less commonly available. The prediction-classification method belongs to unsupervised ML method and can be trained just with data under normal service conditions. Machine learning algorithms based on artificial neural network and autoencoder neural network were developed and evaluated based on simulation data and achieved reasonable accuracy [35, 44, 50]. The accuracy of statistical ML model is dependent upon the uncertainty levels.

To address this important issue, this paper explored data driven machine learning (ML) model for leak monitoring of a desired water area in the WSN. The performance of a data-driven approach is highly dependent upon the availability of historical data. Since data in an actual water distribution network is not widely available at this time, simulated data with industry certified hydraulic model EPANET is used to generate data used in this study. The Artificial Neural Network (ANN), a supervised ML algorithm, and Autoencoder neural network (AE), an unsupervised ML models, were developed to detect leak in a water supply network from sensor data serving a District Meter Area (DMA). Both models, which required balanced or unbalanced datasets respectively, were found to achieve satisfactory results. Strategy to improve detection accuracy is further developed by multiple independent attempts of detection. The article is organized as following: an introduction of the background and methodologies including the theoretical basis of the hydraulic model for water pipe network, background on ML models including artificial intelligence neural network (ANN) and autoencoder neural network (AE), and data generation scheme under both normal and leaking conditions. This is followed by the case studies of two hypothetic water distribution network simulated by EPANET. The performance of ANN, $\mathrm{AE}$ and a postprocessing framework are then described and analyzed by using the first water distribution network. The performance of $\mathrm{AE}$ model is future validated on a larger network with complex water usage scenario, i.e. C-Town water distribution network. Finally, the conclusions are provided to summarize the major discoveries of this paper. 


\section{Theoretical background}

\section{Hydraulic model for water pipe network}

A hydraulic model is commonly used to compute the hydraulic parameters such as water pressure or water head and flow rate for the design of a water distribution network. The governing hydraulic equations describe the conservation of mass and conservation of energy considering the topological characteristics of a water pipe network. The hydraulic model allows to account for the water usage behaviors (described as water demand fluctuations at the service nodes) and events such as leakages on the network performance. While hydraulic model is regarded as sufficiently accurate for water network planning purpose [48], there are uncertainties of the model prediction results due to fluctuating water demands, deteriorating pipe conditions, etc. A calibrated hydraulic model serves as the basis for model-based leak detection. Given it is sufficiently reliable, hydraulic model can be utilized to generate holistic artificial datasets for the development and validation of ML-based leak detection algorithms. As a general note, using holistic artificially generated data is a common strategy in the development of ML technologies when data is not available due to practice constraint. The key equations used for the hydraulic computations are introduced in following.

Equation (1) of the hydraulic model describes the conversation of mass at a pipe node, which prescribes that under no leak condition the inflow of water to a pipe node must be equal to the outflow of water. The outflow of the water including the demand or use of water at that node as well as water flowing from this node to other nodes.

$$
\sum_{p \in P_{n}} q_{p, n}-D_{n}^{a c t}=0, \quad \forall n \in N \#
$$

where $P_{n}$ is the set of pipes connected to the node $n, q_{p}$, ${ }_{n}$ is the flow rate of water into node $n$ from pipe $p\left(\mathrm{~m}^{3}\right)$ $s), D_{n}^{a c t}$ is the actual water demand at node $\mathrm{n}\left(\mathrm{m}^{3} / \mathrm{s}\right)$, and $N$ is the set of all nodes in the pipe network. $q_{p, n}$ is positive when water is flowing into node $n$ from pipe $p$, otherwise, it is negative.

Equation (2) of the hydraulic model describes the conversation of energy. For water pipe network, the total energy is typically referred as the total water head, which includes components describing the kinetic energy (kinetic water head), hydraulic potential energy (pressure head), and gravitational potential energy (elevation head), i.e.,

$$
h_{A}=\frac{u_{A}^{2}}{2 g}+\frac{p_{A}}{\gamma_{w}}+z_{A}=h_{B}+H_{L}=\frac{u_{B}^{2}}{2 g}+\frac{p_{B}}{\gamma_{w}}+z_{B}+H_{L}
$$

where $h$ is the total water head, $u$ is the water velocity at each node, and $z$ is the altitude of each node. $H_{L}$ is the energy loss between node $\mathrm{A}$ and node $\mathrm{B}$.

There are two major mechanisms for the energy lose in a pipe flow [46], i.e., the distributed energy loss and localized energy loss. The distributed energy loss along the pipe due to hydraulic resistance is mainly determined by the velocity of the flow $V$, the internal diameter of the pipe $d$, the length of the pipe $L$, and the roughness of the pipe wall, which is described by the Hazen-Williams formula [26], i.e., Eq. (3).

$$
H(m)=\left(\frac{6.78 L}{d^{1.65}}\right)(V / C)^{1.85}
$$

where $C$ is the roughness coefficient of pipe wall.

The localized energy loss is due to turbulence associated with change of flow conditions (such as flow speed, direction, or flow area etc.), which is determined by the topology of water distribution network connections.

An important phenomenon in a water supply network is the water usage or demand. Two major types of models are generally used for water demand at pipe nodes, i.e., demand-driven model and pressure-driven model. A comparison of both models is described in [6]. A pressure-driven water demand model is used in this study to consider the effects of losing pressure due to change of water demand or leaks.

$$
D=\left\{\begin{array}{cr}
0 & p \leq P_{0} \\
D_{f}\left(\frac{p-P_{0}}{P_{f}-P_{0}}\right)^{\frac{1}{2}} & P_{0} \leq p \leq P_{f} \\
D_{f} & p>P_{f}
\end{array}\right.
$$

where $D$ is the demand at a particular node, $D_{f}$ is the desired $\operatorname{demand}\left(\mathrm{m}^{3} / \mathrm{s}\right), p$ is the water pressure, $\mathrm{p}_{f}$ is the pressure above which the desired demand $D_{f}$ should be met, $p_{O}$ is the water pressure below which no water will be supplied at the node.

The leaking is modeled as a special type of water demand in this study. The demand due to a leaking scenario is related to the size of the leak and is described in Eq. (5) [13].

$$
d_{\text {leak }}=C_{d} A p^{\partial} \sqrt{\frac{2}{\rho}}
$$

where $d_{\text {leak }}$ is the equivalent water demand due to leak $\left(\mathrm{m}^{3} / \mathrm{s}\right), C_{d}$ is the discharge coefficient, with a default value $0.75, A$ is the area of leak, $p$ is the internal water pressure, the exponential $\partial$ is the discharge coefficient, which is 0.5 for steel pipe, and $\rho$ is the water density.

The model is implemented in EPANET, a certified hydraulic model for water supply network (WSN). 


\section{Artificial neural networks (ANN)}

Artificial Neural Network (ANN) is a supervised machine learning (ML) model. The architecture of ANN includes interconnected neurons in the input layers, hidden layers, and output layer. The number of layers and the number of neuron in each layer determine its overall performance [1]. Increasing the number of neurons and hidden layers can improve the ability of ANN model to describe complex nonlinear relationships. It, however, also increase the computational demand and potentially lead to overfitting. An optimal ANN architecture for this study is determined by an optimization process, which leads to an ANN model with one input layer, three hidden layers, and one output layer, as shown in Fig. 1.

The input layer consists of $I$ neurons, which are corresponding to the number of input features. The hidden layers provide the capability to model the complex nonlinear relationships which are fine-tuned with the training data. The output layer consists of one neuron which is used to classify the output as leaking or not leaking condition.

The hidden layers include fully connected neurons, the output of each neuron is written as follows.

$$
y_{k}=f\left(\sum_{r=1}^{I} x_{r, k} \omega_{r, k}+b\right)
$$

where $y_{k}$ is the output of each neuron at the hidden layer, $x_{r, k}$ is the output of the last layer, for the first layer of neural network, $x_{r, k}$ is the sample data $\zeta_{i} . \omega_{r, k}$ is the weight of that neuron and $b$ is the bias of that neuron. The weight and bias are trained with the training datasets by the back-propagation algorithm. $f($.) is the activation function used to increase the nonlinear property during the propagation. In this study, the
'ReLu' function is used as the activation function of the hidden layer, i.e., Eq. (7).

$$
f(x)=\max (0, x)
$$

The output of the last hidden layer is then transferred into the neurons in the output layer, whose actions is written as below.

$$
y_{z}=g\left(y_{k} \omega+b\right)
$$

where and $y_{z} y_{z}$ is the output of the output layer, $y_{k}$ is the output of the neurons in the last hidden layer, $\omega$ and $b$ are the weight and bias. $g($.$) is the tangent sigmoid$ transfer function defined as

$$
g(x)=\frac{2}{1+e^{-2 x}}-1
$$

The ANN model learns the relationship between the output and input by a training process to classify the observed data into leaking and non-leaking situations. More detailed mathematical information about ANN can be found at [19].

\section{Autoencoder neural (AE) networks}

Autoencoder neural network is an unsupervised ML model. It is based on a special type of neural network that is trained to reconstruct its input, so the output $\left(y_{1}, y_{2}, y_{3}, \ldots, y_{n}\right)$ would contain the same information as its input $\left(x_{1}, x_{2}, x_{3}, \ldots, x_{n}\right)$. To reduce the reconstruction error, the network is forced to learn the hidden patterns between the input data. An innovative strategy is proposed in this study to detect the leaking situation by autoencoder neural network based on its reconstruction error. The reconstruction error is characterized by the mean square error:

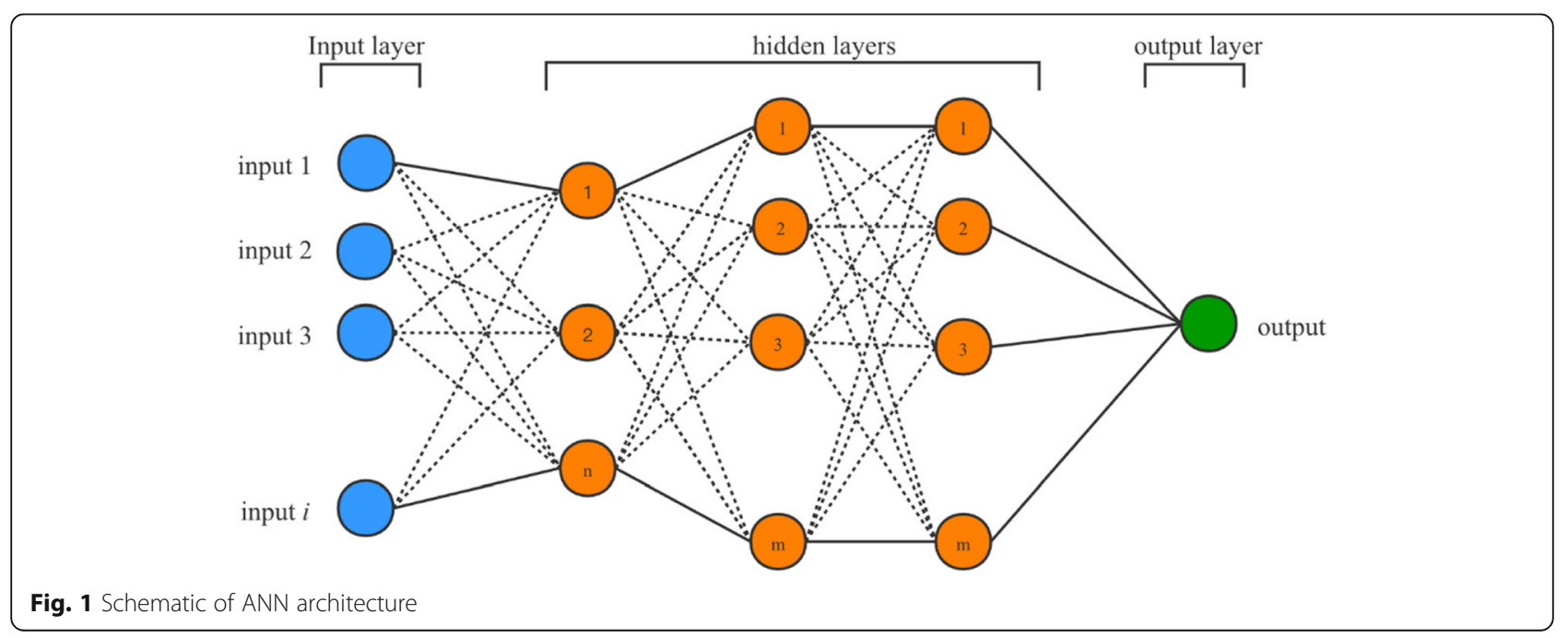




$$
M S E=\frac{1}{n} \sum_{i=1}^{n}\left(x_{i}-y_{i}\right)^{2}
$$

where MSE is the mean square error or reconstruction error, $n$ is the dimension of the input vector $x, x_{i}$ is the sample data and $y_{i}$ is the predicted sample data.

A typical architecture of the autoencoder neural (AE) network is shown in Fig. 2. The training process of the $\mathrm{AE}$ network involves firstly compresses the input vector $x$ into a small dimension, which is called the encoding process. Then the model will reconstruct the compressed data into its original space, which is called the decoding process. By reducing the error between output and input, the weights and bias of the neurons in the neural network are adjusted to learn the relationship among the input data.

For a model trained by dataset of only non-leak condition, a large reconstruction error occurs it is inputted with data of leaking condition because the relationship described by the trained $\mathrm{AE}$ neural network is not valid under such condition. By setting a threshold in the construction error, the AE model can classify if a set of data corresponds to a leaking situation or a non-leaking situation.

\section{Generation of monitoring data in the water supply network under normal and leaking conditions}

Machine learning (ML) model requires a sufficient amount of data for training and validation. When short of real-world data, it is a common approach to develop and evaluate ML algorithms based on simulated data $[18,36,45]$. A python package Water Network Tool for Resilience (WNTR) is used to build the water supply network and solve the hydraulic equations (which is shown in section 2) for water flow in the pipeline system
[23]. WNTR is an open-source python package for hydraulic simulations of water pipe system based on EPAN ET. This package was adopted to run iterative simulations with a combination of a set of random parameters that describes the fluctuation in water demand, data noise, and leaking conditions.

With the WNRT package, WSN operation data (i.e., pressure, flow rates, etc.) with fluctuating water demands, leaking or non-leaking conditions can be generated. The randomness of the WSN model, including water usage fluctuations, pipe conditions and noise, are considered in data generation. The water usage here is an overall estimated during a period. For example, the baseline water usage at each node was chosen from a uniform distribution in the range of 0.008 to $0.012 \mathrm{~L} / \mathrm{s}$ according to Funk et.al [15]. According to [35], the real time water usage may fluctuate from 0.3 times to 1.3 times that of the baseline usage depending on the different time in a day. The pipe conditions are described with the dimensionless roughness coefficient. The roughness coefficient of each pipe was selected from a uniform distribution from 100 to 300. Different levels of noise were also added to account for the uncertainty of WSN such as the water usage or sensor error at a certain time interval and is described with a Gaussian noise $N(0$, $\sigma)$. Similar data generation framework was used by Zhou et.al [50] for a different purpose. The detailed data generation process is introduced in Case study I.

\section{Challenges in leak detection and strategy to overcome the challenges}

There are two primarily challenges in implementing a data-driven model for leak detection of WSN:

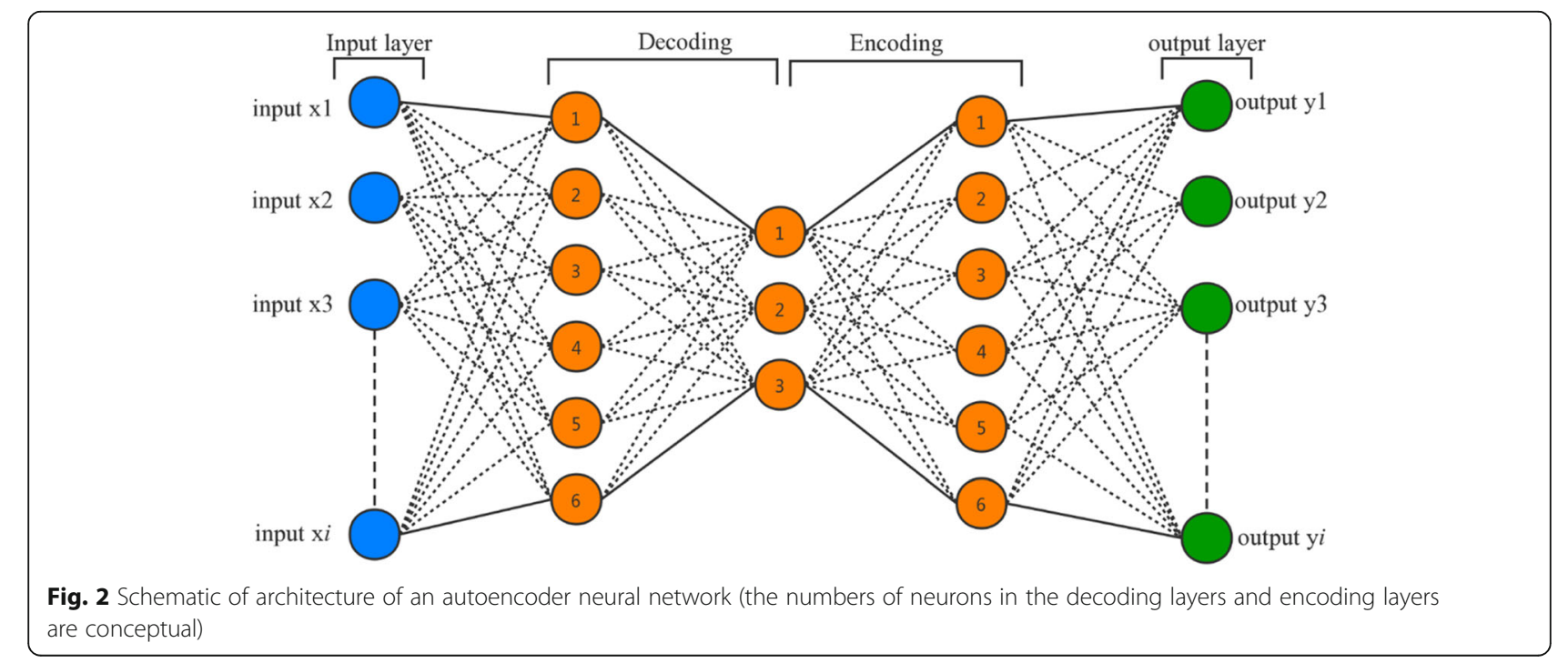


1) Unbalanced data. Many existing leak detection methods, Support Vector Machine (SVM), Convolutional Neural Network -Support Vector Machine (CNN-SVM), ANN, etc., treat leak detection as a classification problem $[9,20,31]$. These methods require a sufficient large leaking dataset as well as non-leaking datasets. However, as pointed out by Mounce et.al [30, 32], there are relatively limited leaking situations compared with nonleaking situation. Let alone to require data of leaking occurring at each pipe of a water distribution system. This leads to overly unbalanced datasets, i.e., much more non-leaking data than leaking data. A major challenge of a data driven leak detection approach is how to use little or no data collected under leaking condition to train ML model for leak detection.

2) Uncertainty of user demands. The water pressure pattern in the service water pipe network may be unstable due to the fluctuation of water use behaviors [48]. The water use in the water supply network is strongly affected by the user behaviors and can show strong fluctuations. The predictionclassification method has been developed for this purpose [48]. For example, methods by $[1,10]$ predicted the water pressure at the next time step by different methods. Baker et.al used an adaptive forecasting model to predict the short term water demand of a DMA [4]. Leaking alert is triggered if the difference between the actual water pressure and predicted water pressure exceeded a threshold. Water flow data at midnight when user water demand is small has been used to identify the leaks, under the assumption that baseline flow at midnight is most likely due to leaks [29] when fluctuation in user demand is small. However, most of these detection methods require a consistent or predictable trend of water demand, otherwise it will trigger false alarms. The spatial relationship of multiple nodes in the water distribution network can be used to mitigate false alarms in leak detection. For example, Zhou et.al [50] used a full linear DenseNet neural network with the spatial information of multiple sensors in a water distribution network for leak detection. However, such spatial information was only used in supervised learning which requires sufficient amount of data under leaking conditions and therefore present challenges as stated in item 1).

To address these two challenges, a novel leak detection method is proposed in this paper based on both the spatial and temporal information. The spatial pattern among a group of nodes is used in leak detection and identify leak conditions. The temporal information is used to further improve the detection accuracy. The advantage of the new leak detection model is that it can be trained with data under non-leaking conditions only.

\section{Case studies of ML models for leak detection in water supply network}

Two water supply networks (WSN) were analyzed to illustrate the proposed data generation framework and develop new leaking detection methods. The first network is a relatively small WSN that has been widely used as a standard testbed, which was chosen to illustrate the data generation process, development and validation of proposed leak detection methods. The second network is a large size WSN containing 5 district meter areas (DMA), multiple water sources ( 7 tanks and 1 reservoir) and 6 different control rules (such as valve controls and pump controls). The second WSN network was used to demonstrate the performance of the developed leak detection method under more complex conditions.

\section{Case study - I: rancho Solano zone III water distribution system}

Rancho Solano Water Network located in the city of Fairfield, California. This network is published by ASCE task committee on a research database for water distribution systems [17]. The graph of this water supply network is shown in Fig. 3. There are 112 nodes in total, including one reservoir as the source of water, and 126 pipes. The elevations of the nodes in this pipe network range from $90 \mathrm{~m}$ to $120 \mathrm{~m}$ and the length of the pipes range from $90 \mathrm{~m}$ to $130 \mathrm{~m}$.

The water distribution network and basic water demand at each service node are shown in Fig. 3. The basic demand of each node is chosen from a uniform distribution of 0.008 to $0.012 \mathrm{~L} / \mathrm{s}$. The real demand at each node is generated by adding a random Gaussian distribution with variance $\sigma=0.01 \mathrm{~L} / \mathrm{s}$. Eleven demand ratios from 0.3 to 1.3 are considered during the data generation with the hydraulic model for the WSN. The monitoring sensors are assumed to be deployed in the area shown in the red circle area, i.e., the water pressure data of the nodes which are located in the red circle in Fig. 3 is used for leak detection. Figure 3 also shows some key nodes and pipes that are analyzed in the study.

The pressure-driven demand model, which relates the water discharge to the water pressure head at the node (i.e., Eq. 4), is used in the hydraulic analysis of the WSN. The lower bound of the pressure head at the node is set as $5 \mathrm{~m}$ and the upper bound as $30 \mathrm{~m}$. An example of the relationship between demand/discharge and pressures head at a node with base demand of $0.02 \mathrm{~m}^{3} / \mathrm{s}$ is shown in Fig. 4.

The overall data generation procedures of balanced dataset with the hydraulic model for the WSN are briefly 


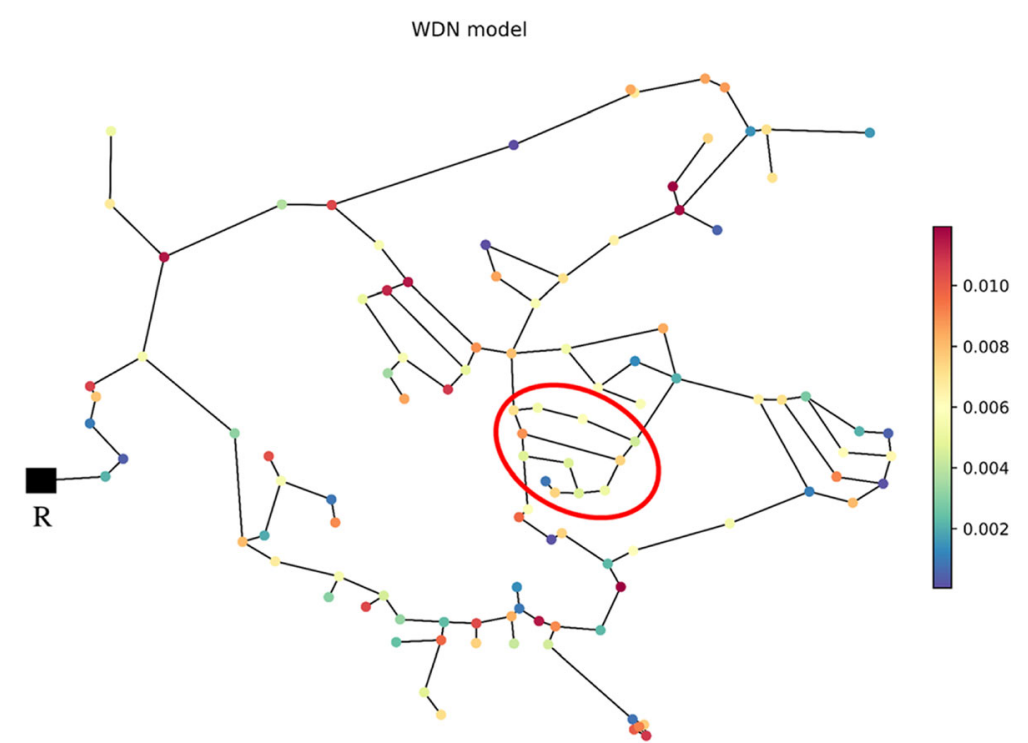

Fig. 3 Illustration of the water supply network and water demand at each node (color code corresponds to basic water demand in the unit of $L / S)$

summarized below. Similar amount of data is produced for the monitoring area in the WSN under both leaking and non-leaking conditions.

\section{Dataset for non-leaking conditions}

1. Define Water Pipe Network: Construct the pipe network according to Rancho Solano Water Network (Fig. 3) using EPANET data input format.

2. Assign Water Pipe Conditions: Assign the pipe roughness of pipe with a random number from uniform distribution $U(100,300)$. The length of each pipe is already defined in the original water pipe network
3. Set the Baseline Demand and Actual Demand at User Nodes: the baseline demand of each node is randomly selected from a uniform distribution $U(0.008,0.012) L / s$, following Funk et al. [15]. The actual demand at each node is set by considering both the base demand and the demand uncertainty, i.e.,

$$
\text { Demand }=\text { deman ratio } * D_{\text {base }}+|N(0, \sigma)| .
$$

where $D_{\text {base }}$ is the predefined base demand. The demand ratio is set from 0.3 to 1.3 to account for the fluctuation

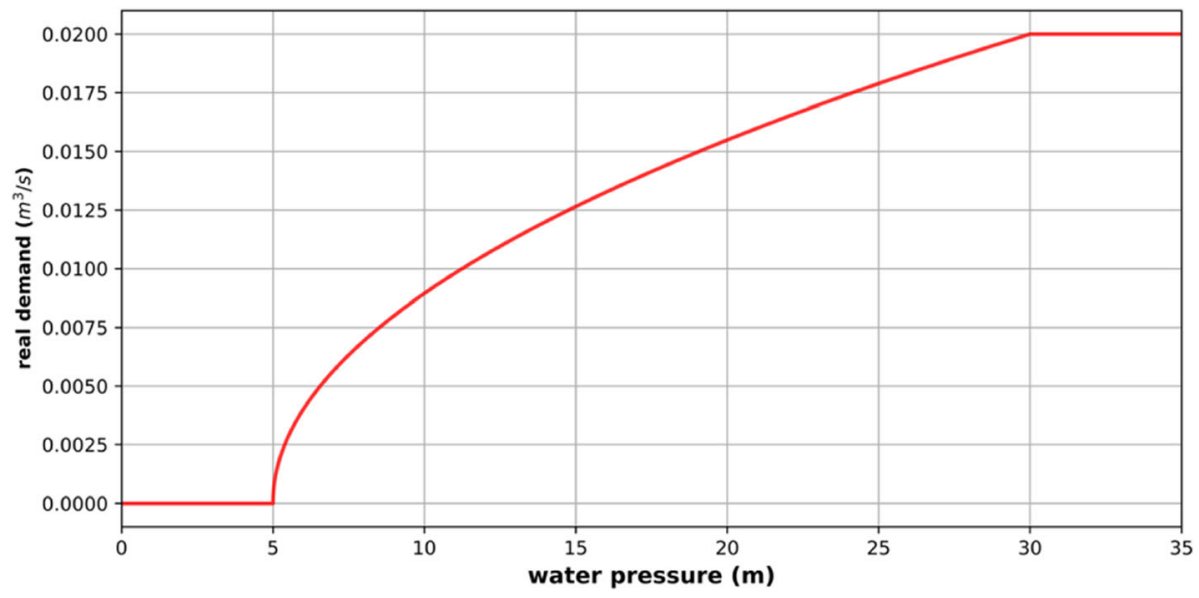

Fig. 4 Example of the relationship between water demand and pressure head at a node with base demand of $0.02 \mathrm{~m}^{3} / \mathrm{s}$ 


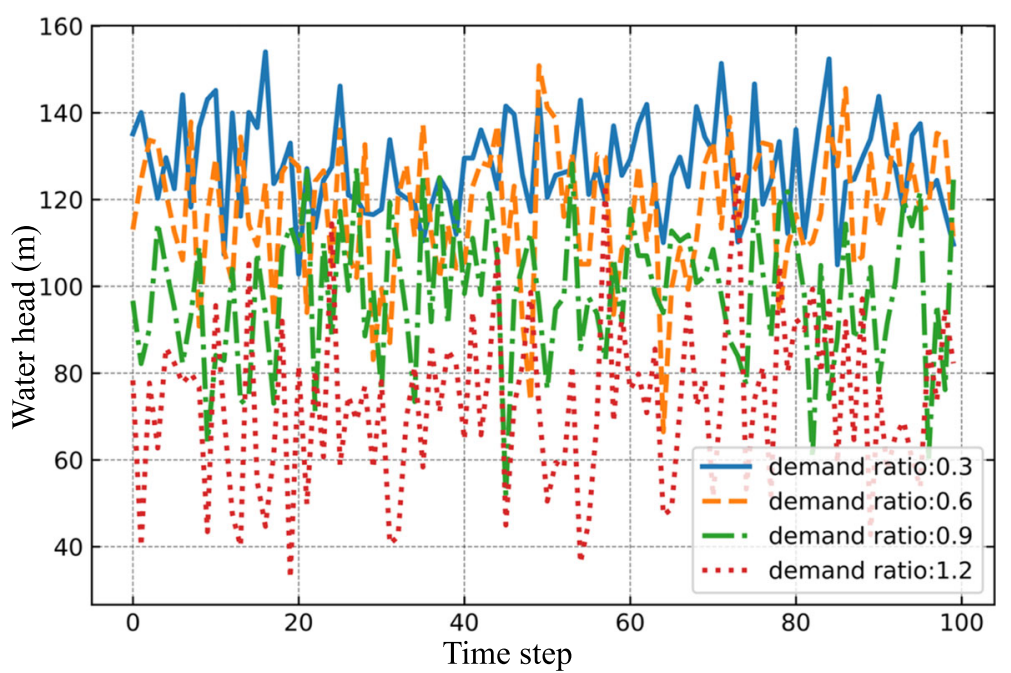

Fig. 5 Example of water head at node ' 168 ' under different water demands (note: demand ratio is defined as the average water demand to the predefined baseline demand at the node (Eq. 11); the fluctuation is due to fluctuations in the real demand)

in water usage demand during a day or between different days. Gaussian noise $N(0, \sigma)$ considers the uncertainty due to the water usage fluctuation.

4. Data Generation: solve the hydraulic model of the WSN with WNRT using the EPANET built-in module and record the water pressure at selected monitoring nodes (i.e, the nodes inside the red circle in Fig. 3).

5. Data generation for different water demand situations: step 2 to step 4 are repeated for each water demand scenery. 200 rounds of simulations were conducted for each scenery to generate sufficient amount of data under different water demand situations,

\section{Dataset for leaking conditions}

1. Similar procedures as for non-leaking conditions are followed to build the water network, assign pipe roughness and water use demands (Step 1-3 for non-leaking conditions).

2. Leak Scenario: Set pipe $i$ as the leaking pipe. By default, the leaking position is located at the middle of the pipe, which, however, can be easily changed for more complex scenario.

3. Data generation: solve the hydraulic simulator with WNRT using the EPANET built-in module and record the water pressure at selected monitoring nodes (inside the red circle in Fig. 3).

4. Data generation for different water demand situations: Repeat steps 2-4200 times for each pipe at demanding level similar as what is done for nonleak conditions.
5. Repeat the above step for each pipe leaking scenario.

The water pressure data under different scenarios were generated via the processes described. The nonleaking situation and leaking situation at each pipe contain 2200 cases respectively (11 different water demand levels with 200 rounds of simulations). It is noted that the model-generated data can be easily replaced with real-world data when measurement data is available.

The code for data generation is published in this link for the sake of open source. ${ }^{1}$ Overall, the water pressure is affected by the average demand at the node, fluctuations in water demand, and if leak occurs. To illustrate the characteristics of water pressure data, the water pressure of node ' 168 ' under a few demand ratios are shown in Fig. 5. As can be seen, the water pressure can be highly influenced by the demand levels. There are significant differences in the water pressure between demand ratios of 0.3 versus 1.2. A higher water pressure corresponds to a lower water demand. It should be noted that water pressure is also affected by leak. For example, Fig. 6 shows the water pressure at node ' 168 ' is similar for intact water pipe at high average water demand ratio of 1.0 versus leaking pipe (pipe 198) with low average water demand ratio of 0.3 . Such overlap in the influence on water pressure by water demand and leaking makes it difficult to detect leak from data from a single node. Machine Learning model, however, allows to extract features from the spatial pattern in the pressure data

\footnotetext{
${ }^{1}$ https://github.com/herewego321/Random-WDN-data-generation
} 


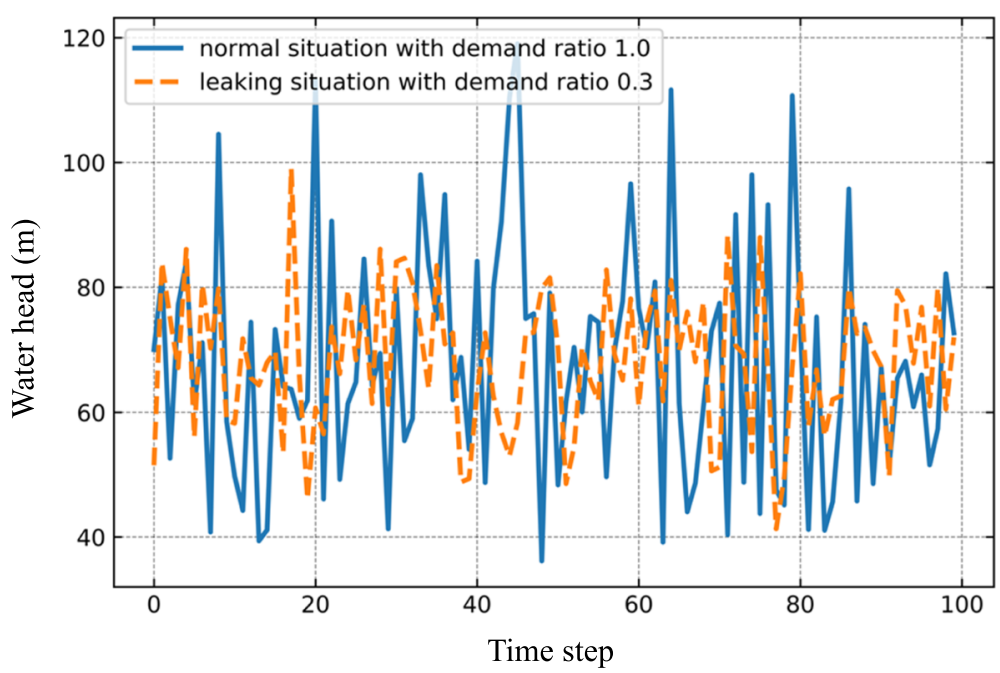

Fig. 6 Illustration of water head at node '168': the average water head is similar for non-leaking condition with high water demand versus leaking condition with low water demand

at multiple nodes and therefore allows to differentiate leaking versus non-leaking conditions.

\section{Artificial neural network (ANN) model for leak detection}

An ANN model is developed to detect leaks. The water pressure data at a group of monitoring nodes is used for this purpose. Unlike the existing approach of using time series analyses, the water pressure data are used by ANN model to find the spatial relationship among data at monitoring nodes at a given time. The ANN model was built and trained with TensorFlow in python environment. An optimal ANN architecture for this study is determined by an optimization process, which leads to an ANN model with one input layer, three hidden layers, and one output layer. The input layer contains 11 neurons corresponding to the 11 monitored nodes. The first hidden layer contains 128 neurons and then the remaining two hidden layers contain 258 neurons respectively. The output layer contains 1 neuron, which is a categorical data indicating leaking versus non-leaking condition.

Overall, the ANN model is used to classify leaking and non-leaking conditions of the monitoring area as a binary classification problem. As a supervised learning model, ANN requires the dataset to be labeled prior to training. A data set contains 2400 samples is prepared for ANN training process, which includes 1200 nonleaking samples and 1200 leaking samples. The nonleaking samples are randomly selected from the simulated non-leaking dataset and labelled as 0 . The leaking samples are randomly selected from the leaking dataset that contains simulated data when leak occurred inside the monitoring area and are labelled as 1 . The leaking and non-leaking dataset are generated follow the procedures that described previously. Standardization of the dataset is conducted to reduce the computing time and avoid potential overfitting. Each row of the dataset is transformed to a normal distribution with zero mean and unit variance. The benefits of data standardization is described by [40]. The 2400 labelled dataset is then randomly split into independent training data and testing data with a ratio of 7:3 (i.e., 1680 set of training data and 720 set of test data). The training dataset is used to train the ANN model. The independent testing data is used to validate the model results. The loss value of training and validation processes are shown in Fig. 7 . The loss value is the mean square error of predicted result versus actual result. As can be seen, both loss values of training dataset and validation dataset decrease to small values during the learning process, which means the ANN model is

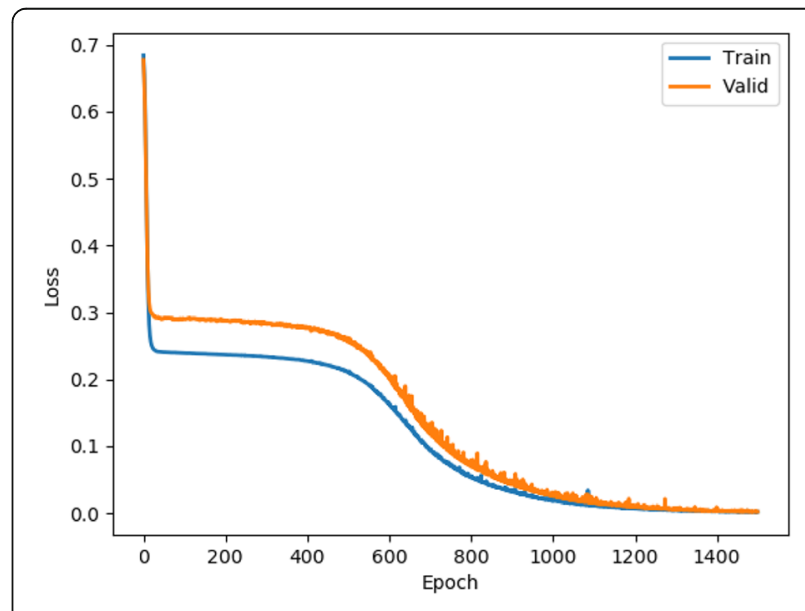

Fig. 7 Loss values during the ANN model training process 
able to uncover the relationship among data for classification of leak versus non-leak conditions.

The final classification result by the trained ANN model using the testing data is shown in Fig. 8 as described with the confusion matrix. There were 370 nonleaking cases and 350 leaking cases in the testing dataset, both are classified with $100 \%$ accuracy and with no misclassification. The results imply that a) the relationship of water pressure among a group of nodes is different under leaking and non-leaking scenarios; and b) the ANN model is trained to extract this relationship and to accurately classify leaking versus non-leaking conditions.

\section{Autoencoder neural (AE) network model for leak detection}

The ANN model achieved excellent performance by utilizing the water pressure data at multiple nodes. However, as a supervised ML model, ANN model requires balanced data, i.e., similar amount of data under both normal and leaking conditions. However, in the reality, the available data is typically unbalanced. i.e., there might be only limited amount of data under leaking conditions compared with data under non-leaking conditions. Besides, labeling the dataset to leak or non-leak conditions, i.e., such as the method used by [50] may be extremely difficult under real situation since the leaks might not be detected until their effects surface.

A variation of ANN model, the autoencoder neural (AE) network, is developed for leak detection to resolve the challenge of unbalanced data. As an unsupervised ML model, the AE model features unique advantages to work with unbalanced data. In this study, a AE model

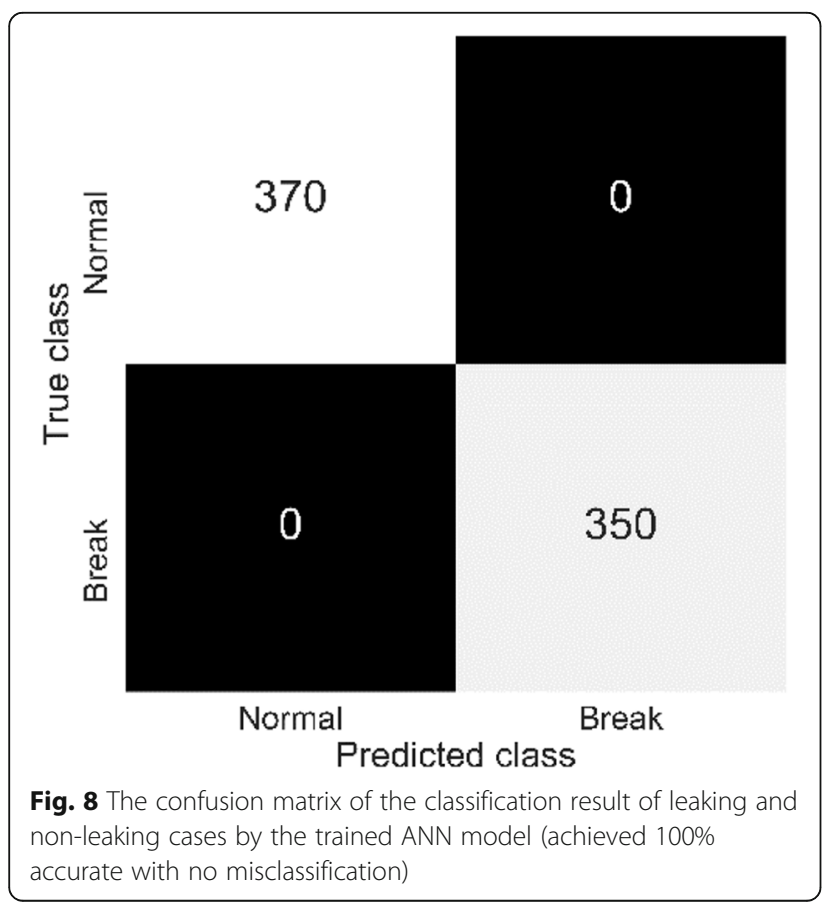

with 5 layers was built. As shown in Fig. 2, the first and last layer contains 11 neurons which are corresponding to the 11 monitored nodes. The second and third layer encodes the input data from 11 nodes to a lowerdimensional space, while the fourth and fifth layer decodes the data from this lower-dimensional space back to 11 nodes. The hidden layer of the AE model contains 3 neurons. Since the AE model features the ability to detect abnormal samples from dataset of normal samples, only the non-leaking samples are used for the training purpose. One thousand two hundred samples are randomly selected from the non-leaking dataset to train the AE model. Each sample in the dataset includes the water pressure information at the 11 selected monitoring nodes. $70 \%$ of the 1200 normal non-leaking dataset is standardized and used for training. The rest $30 \%$ of the normal non-leaking dataset is used for validation.

Figure 9 shows the loss values, defined as the reconstruction error for AE model, during the training process. Small loss values of close to zero are achieved for both the training and testing data, meaning the reconstructed output from the AE model is close to its input of dataset under normal non-leaking conditions. This also implies that the AE model is well trained with the normal non-leaking dataset. When leaking dataset is input to the trained AE model, the model will generate a large reconstruction error, which can be used to detect leaking conditions.

Independent datasets are used to evaluate the performance of the trained AE model to detect leaking conditions. The dataset includes three different scenarios, i.e. Five hundred fifty datasets from the non-leaking conditions, i.e. there is no leaks anywhere in the water distribution system; 550 cases of dataset where a leak happens at a random pipe inside the monitoring area (pipe '163'); 550 cases of datasets where the leak happens at a random pipe outside the monitoring area ("pipe '198'). The location of example pipes can be found in

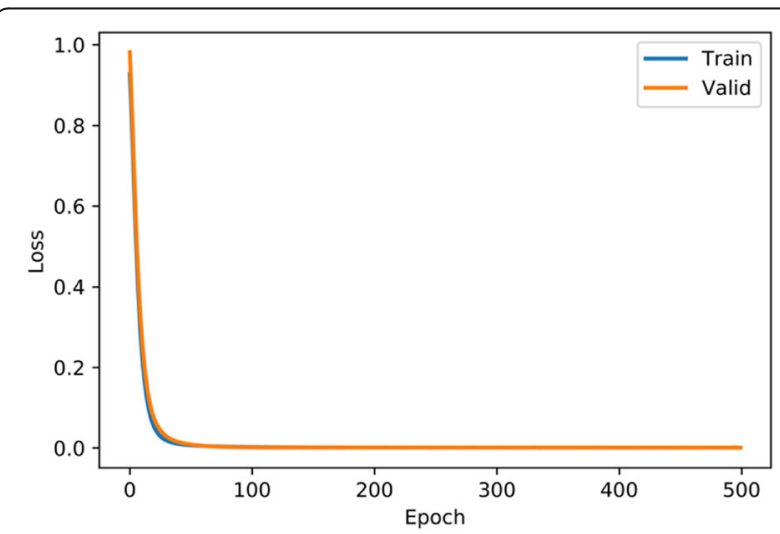

Fig. 9 Loss values (i.e., the reconstruction error) by the AE model during the training process 
Fig. 3. All of the data samples are normalized based on the mean and variance values of non-leaking dataset (by minimizing the mean value and divided by the variance value).

Figure 10 shows the statistics histogram of the reconstruction errors by the $\mathrm{AE}$ model for data under the three different scenarios. The rectangles with different color lines indicate the $97.5 \%$ range of reconstruction error for normal and two leaking situations. As can be seen in Fig. 10, the reconstruction error of data under normal non-leaking situation is small, with $97.5 \%$ of reconstruction error less than 0.00015 , which is much smaller than those under the other two situations. The reconstruction error of data when leak occurs inside the monitoring area features largest reconstruction errors. While the reconstruction errors of data for leaking outside the monitoring area lies in between. Overall, dataset corresponding to pipe leaking within the monitoring area leads to large reconstruction error by the trained AE neural network model. The differences in the reconstruction error are clearly differentiable from those by the normal non-leaking cases. This can be used to define the threshold for leak detection. Leaks occurring outside the monitoring areas, however, still has a low probability to be identified as leaking situation. It should be noted that since a certain area is monitored by these sensors, the ideal result is only the leaking inside or very closed to this area can be detected. Leaking faraway from this monitoring area should not be able to trigger the detection. Method to mitigate detection error due to the influence of leaking outside the monitoring areas will be discussed in next sections.

Based on the observation, a threshold of reconstruction error can be defined for the trained $\mathrm{AE}$ neural network can be used to differentiate the leak versus non-leak situations. This threshold can be set based on the training process and be further tuned when data under leaking conditions is available. A threshold of 0.000402 is set for this case based on the reconstruction error at the end of $\mathrm{AE}$ neural network training. With this trained $\mathrm{AE}$ model, the monitored water pressure data can be fed into the trained model to obtain the reconstruction error. If the reconstruction error is larger than the set threshold value, a leaking alert would be triggered to promote actions such as inspection and replacement.

The performance of $\mathrm{AE}$ is evaluated at the situations where leaking happens at each pipe. For each single pipe, independent data of 2200 non-leaking cases and 2200 leaking cases are generated. The water pressure data inside the monitoring area is then fed into the trained AE model. Fig. 11 summarizes the probability of leaking alert is triggered, i.e. percentage of cases with a reconstruction error larger than the threshold, under each pipe leaking conditions of the WSN. As can be seen from Fig. 11 a), the alert triggering probability (i.e., false alert) under non-leaking situation is very low, or only about $3 \%$ maximum. Fig. 11 b) shows the probability leaking alert is triggered when leak occurs at each pipe. For the leaking happens inside the monitoring area, the alert has $68 \%$ to $100 \%$ probability to be triggered. For leak happens outside the monitoring area, the chance of triggered the alert is compromised (less than $40 \%$ for most parts). These observations imply that $\mathrm{AE}$ model can detect leaks from the monitored water pressure data. For the globally monitoring purpose, the monitoring sensors need to be strategically deployed in the WSN to achieve high reliability in leak detection.

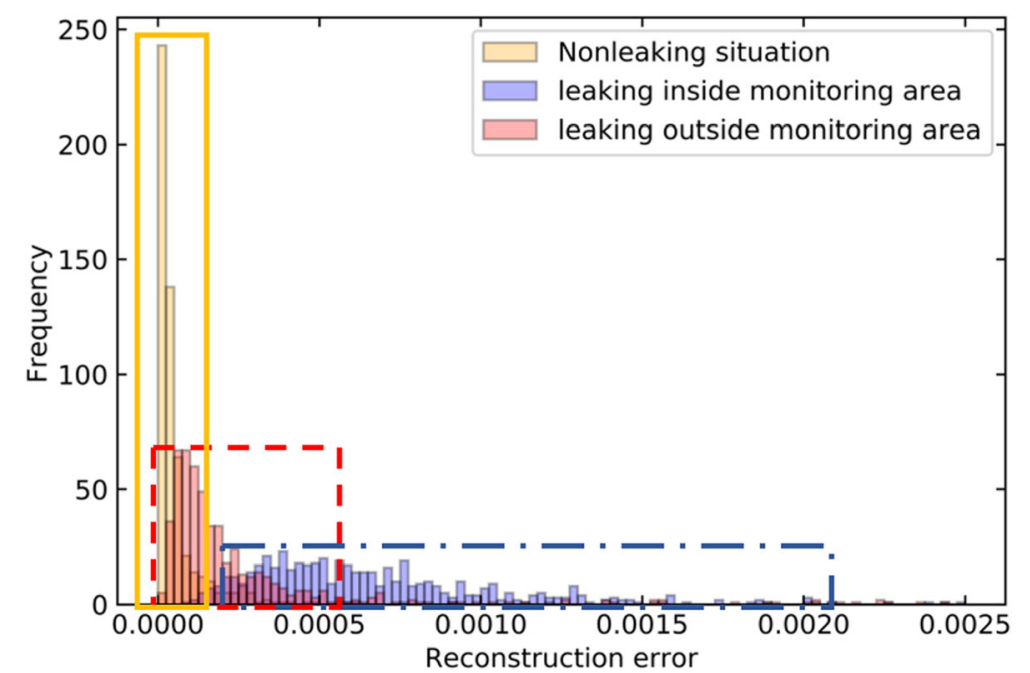

Fig. 10 Reconstruction error by AE model for data under normal non-leaking condition versus leaking situation (including leaks inside and outside the monitoring area) 


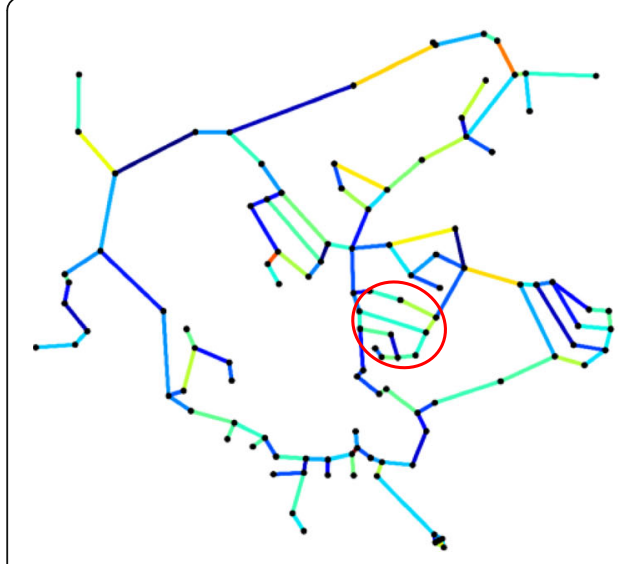

a)
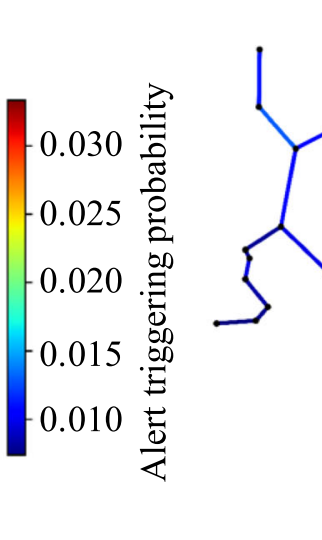

Fig. 11 Probability of AE model triggers leak by individual water pipe in the WSN under a Non-leaking condition, $\mathbf{b}$ Leak condition (encircled are the monitoring area where water pressure data is collected)

Sensitivity study on factors affecting the accuracy of the $A E$ model in leak detection

Sensitivity study is conducted to evaluate the effects of contributing factors on the performance of $\mathrm{AE}$ model for leak detection. These include three independent factors, including the compression ratios of $\mathrm{AE}$, the sizes of leak, and fluctuation/uncertainty of water demand.

The compression ratio is the number of uncompressed data divided by compressed data as calculated in Eq. 12. It is an important hyperparameter of the AE neural network. A large compression ratio can not only save the physical data storage space but also force the AE model to learn the internal pattern of input data. However, too much compression may lead to excess information loss and decrease the detection accuracy. The range of compression ratio is selected between 1 to 6 for the sensitivity study.

$$
\text { Compression Ratio }=\frac{\text { Uncompressed Size }}{\text { Compressed Size }}
$$

The uncertainty of water usage is the description of water demand fluctuation during a day. A higher fluctuation of water demand increase the difficulty for leaking detection since water demand and leak both affects water pressure in the WSN. To describe its sensitivity, the uncertainties of water usage are assumed to follow a normal distribution and are described with different water usage uncertainty levels, i.e., $\mathrm{N}(0,0.001) \mathrm{L} / \mathrm{s}$, $\mathrm{N}(0.005) \mathrm{L} / \mathrm{s}, \mathrm{N}(0,0.01) \mathrm{L} / \mathrm{s}, \mathrm{N}(0,0.05) \mathrm{L} / \mathrm{s}$.

The leaking size is another important factor that influences the detection system performance. Conceptually, detection of small leak is much difficult than large leak, since smaller leak has less influence on the status of WSN and can be inundated with water demand fluctuations. For the sensitivity study, the leaking size is varied from $0.01 \mathrm{~m}$ to $0.12 \mathrm{~m}$.

For each combination of these three factors, the performance is evaluated by a dataset generated by assuming leak occurs in a pipe (pipe '163') inside the monitoring area and a data set with non-leaking. The data is randomly split for independent validation. The final accuracy is calculated as the average accuracy from a 3 rounds of crossvalidation processes. Fig. 12 shows the leak detect accuracy of the AE models affected by the compression ratios, water usage uncertainty, and leak sizes.

As shown in Fig. 12, the AE model achieved close to $100 \%$ accuracy when uncertainty with water usage is small. At a given leaking size, the accuracy of leak detection by the $\mathrm{AE}$ model decreases with the increasing water usage uncertainties. As the water usage uncertainty level increases from $0.001 \mathrm{~L} / \mathrm{s}$ to $0.015 \mathrm{~L} / \mathrm{s}$, the accuracy of the model decreases from 100\% to $89.93 \%$. However, even with high variance in water use uncertainty (compared to the baseline water usage at $0.012 \mathrm{~L} /$ $\mathrm{s})$, the AE model achieved decent accuracy in leak detection.

The performance of AE model is significantly influenced by the leak size. Small leaks tends not to be detected and WSN is classified under normal non-leaking situations (i.e., $0 \%$ correct detection). While normal nonleaking cases are all classified correctly (i.e., 100\% correct detection). This gives an accuracy of around 50\% for a balanced dataset with equal number of data under both leaking and non-leaking conditions. With increasing leaking sizes, the AE model achieved higher leak detection accuracy. This is reasonable since the larger the leak size, the more disturbance it will have on the pressure distribution in the WSN to allow its detection. A similar conclusion was shown in Zhou et.al [50]. 


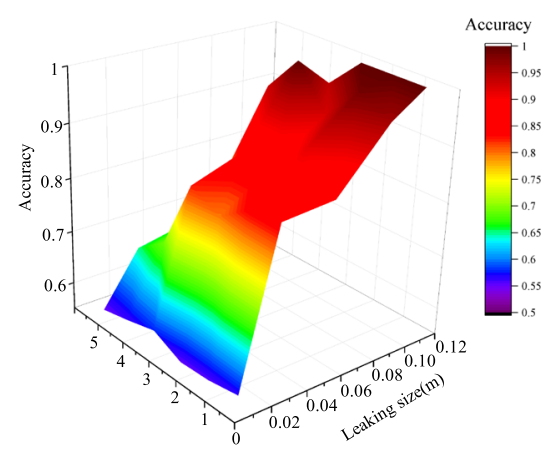

a)

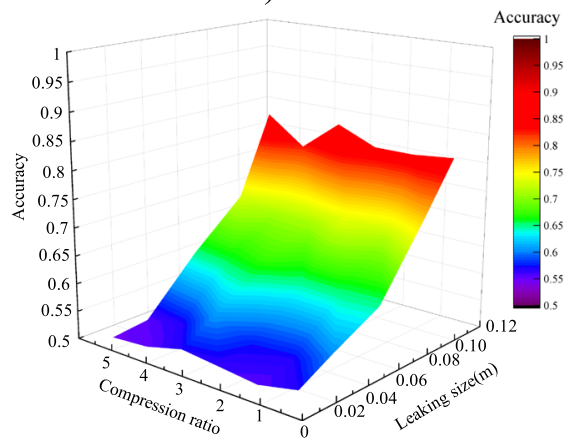

c)

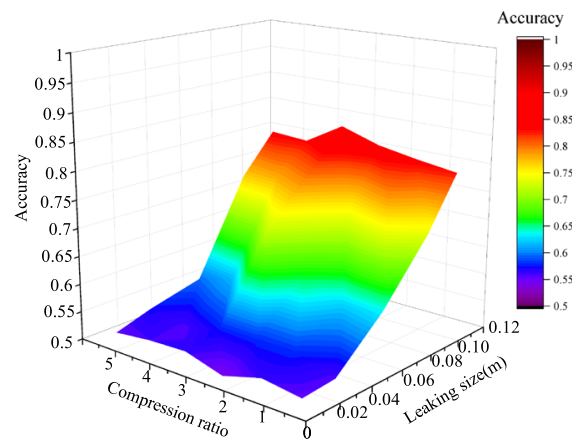

b)

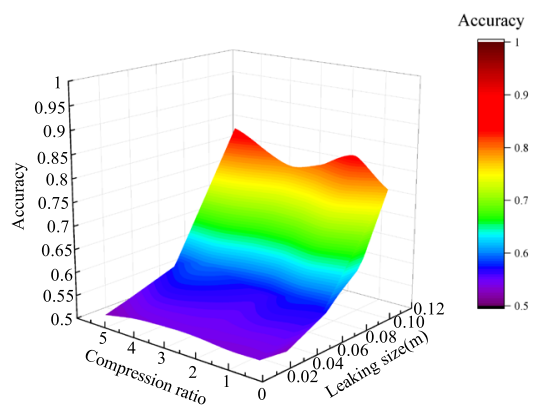

d)

Fig. 12 The sensitivity of leak detection accuracy by AE model on the compression ratio, leak size, and water usage uncertainty: a Water usage uncertainty of $\mathrm{N}(0,0.001) \mathrm{L} / \mathrm{s}$; water usage uncertainty of $\mathrm{N}(0,0.005) \mathrm{L} / \mathrm{s}, \mathbf{c}$ Water usage uncertainty of $\mathrm{N}(0,0.01) \mathrm{L} / \mathrm{s}, \mathbf{d}$ Water usage uncertainty of $\mathrm{N}(0,0.015) \mathrm{L} / \mathrm{S}$

The compression ratio has a negative influence on the overall detection accuracy. For example, as shown in Fig. $12 \mathrm{a}$ ), at the leaking size of $0.06 \mathrm{~m}$, the accuracy decreased from $85.24 \%$ to $67.02 \%$ when compression ratio increases from 1 to 6 . For leaking size of $0.11 \mathrm{~m}$, the accuracy decreases from $100 \%$ to $80.75 \%$ when compression ratio increases from 1 to 6 . This is reasonable since the higher compression ratio will loss more information of original dataset. However, it is also noticed that the influence of compression ratio is small for compression ratio less than 2. A compression ratio of around 1.5 appeared to achieve the best results. It also should be noted that compared to the other two factors (leaking size and water demand uncertainty), compression ratio has a relatively smaller impact on the detection accuracy. However, for a given leak size and water demand uncertainty, fine tuning the compression ratio of $\mathrm{AE}$ model helps to achieve a higher detection accuracy.

\section{Improving classification accuracy by incorporating multiple independent detection}

The previous results show that by setting the proper detection threshold, the AE model achieved good leak detection accuracy using unbalanced data. This is a major advantage to the conventional ANN model, which requires balanced data under both non-leaking and leaking conditions. An observation is that the AE model did not achieve as high accuracy as the ANN model. It is desirable to further improve the accuracy of AE model that will help to reduce the amount of false detection (i.e., false leaking alarm or missing detection of leak event) detection. A method is proposed to further increase the leak detection accuracy by utilizing the probability theory for multiple independent trials. Intuitively, since leaking in the physical world will last for a while before it is repaired, the chance to detect the leak is higher if the effort is attempted multiple times. The leak status is unveiled by a voting strategy. In other words, for $n$ attempts in leak detection, the detection outcome is defined as the outcome by the majority (more than 50\%) of these attempts. Since each detection attempt is via independent data set, each represents an independent trial. Mathematically, if the probability of correctly detecting a leak under a single attempt is $\mathrm{p}$, then the probability of more than half attempts correctly detect the leak will be

$$
\mathrm{p}=\sum_{i=\operatorname{int}\left(\frac{n}{2}\right)}^{n} C_{n}^{i} p^{i}(1-p)^{n-i}
$$


where $\mathrm{n}$ is the number of the total attempt for identifying a leakage. $\mathrm{C}_{\mathrm{n}}^{i}$ is the set of $i$ combination of set $n . n$ is the total number of monitoring cases. $p$ is the correct detection probability of each case.

According to Eq. (13), the probability of correct detection approaches to 1 when $n$ approach infinite $n \rightarrow \infty$, under the condition $\mathrm{p}$ is larger than 0.5 .

According to the principle described by Eq. 13, multiple attempts were made for leak detection, i.e., multiple datasets under a given leaking or non-leaking condition are fed into the AE leak detection model. The final designation of leaking or non-leaking condition is based on if more than half of the detection attempts give that result.

To evaluate the performance of multiple attempts, three scenarios are considered, i.e. non-leaking situation, leaking in pipe 163 located inside the monitoring area, and leaking in pipe 214 located outside the monitoring. Two thousand two hundred set of pressure data are generated under each scenario. For each scenario, the accuracy with $\mathrm{n}$ times of attempts is calculated by the following procedures (also illustrated in Fig. 13):

1) n sets of data are randomly selected from the 2200 cases.
2) Each of the dataset is fed into the AE model to generate an output of Leak or Non-leak condition based on the set threshold.

3) The final designation of Leaking versus NonLeaking condition based on more than half of the $n$ attempts give that condition.

4) Determine if the defection is correct or wrong by comparing the detected condition by 3 ) with the actual condition of the pipe.

The procedure from 1) to 4) are repeated 1000 times. From this, the overall accuracy in correctly detecting the pipe condition is calculated.

Effects of multiple attempts The results of accuracy under multiple attempts of detections using the pretrained AE model are shown in Fig. 14. The detection threshold is set as 0.000402 . The vertical axis is the detection accuracy of the pipe condition. The horizontal axis indicate the number of attempts in detection. As seen from this figure, the detection accuracy improved with multiple attempts and achieved close to $100 \%$ detection accuracy, regardless where leak occurs. This is consistent with what is predicted by Eq. 13 .

Effects of detection threshold by the AE model Threshold is a critical parameter for the AE leak

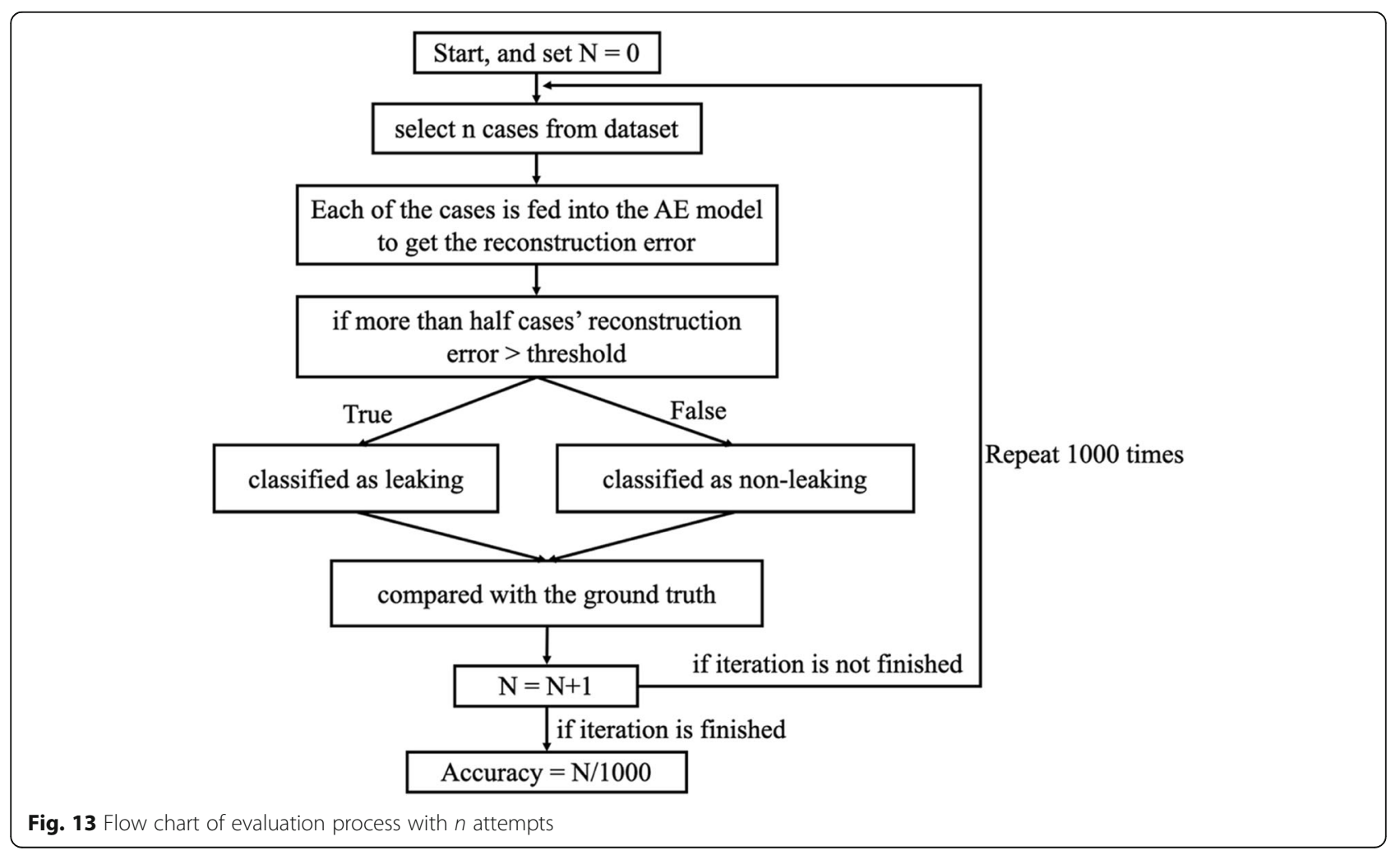




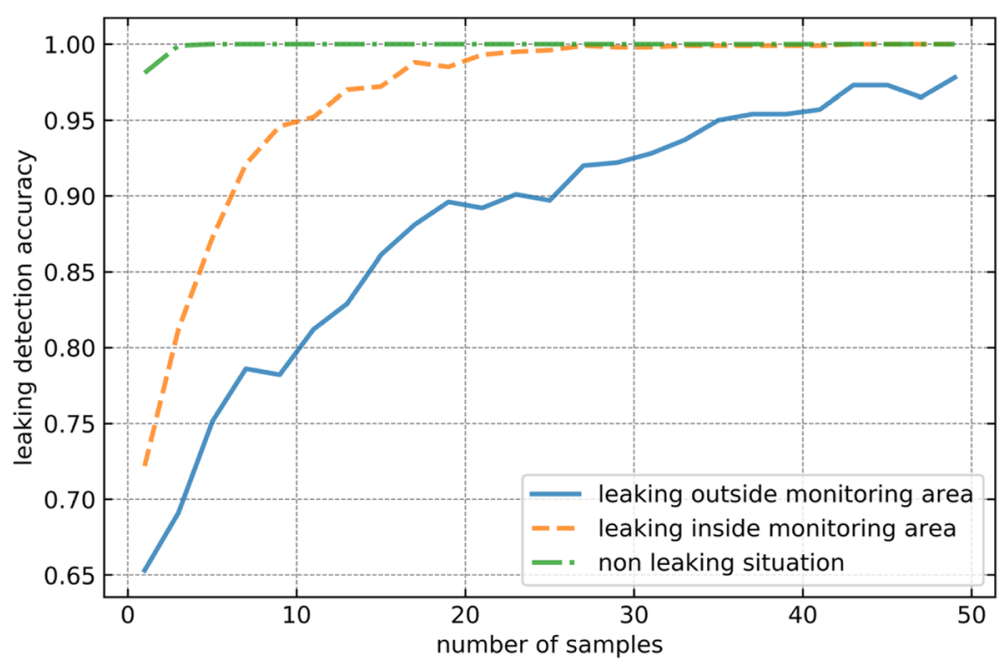

Fig. 14 Accuracy of correct pipe condition detection with multiple attempts with pre-trained AE model with threshold set as 0.000402

detection model. Sensitivity analyses are conducted on the influence of threshold on the model detection accuracy. The results for the three different scenarios defined as in the previous context are shown in Fig. 15. Two thousand two hundred cases of dataset were generated for each scenery and are fed into the AE model to determine the reconstruction errors. From this, the percentage reconstruction error by $\mathrm{AE}$ model larger than the reconstruction error is determined. The horizontal axis are the thresholds and vertical axis is the percentage of cases with reconstruction error larger than the threshold (i.e., the case is identified as leaking by the AE model). The $50 \%$ line is also indicated in the figure. As can be seen from the figure, for all the three pipe condition sceneries, a smaller threshold corresponds to larger chance for the condition to be identified as leaking condition. For no leaking condition, this presents as false alarm. A larger threshold reduces the false alarm but may miss leaking cases. According to the eq. 13, leaks can be properly identified with multiple attempts as long as a detection accuracy is larger than 0.5 . Based on this criteria, leak within the monitoring area can be accurately detected with any threshold between 0.00029 to 0.00057 , by use of the multiple attempts strategy.

By using the proposed post-processing method with 50 time steps data. The final detection result is shown in Fig. 16. The result clearly demonstrates that when leaking happens inside or nearby the monitoring area, the $\mathrm{AE}$ model is able to detect such leaking happens correctly. For leaking which is far away from the monitoring

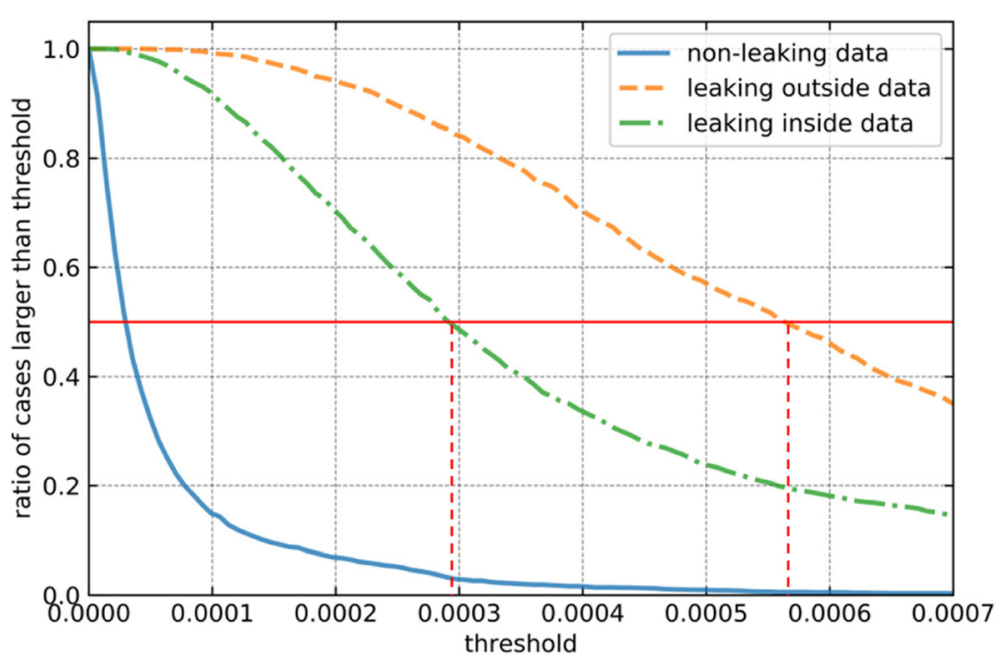

Fig. 15 Percent of leak warning at different detection thresholds of AE model under different pipe conditions 


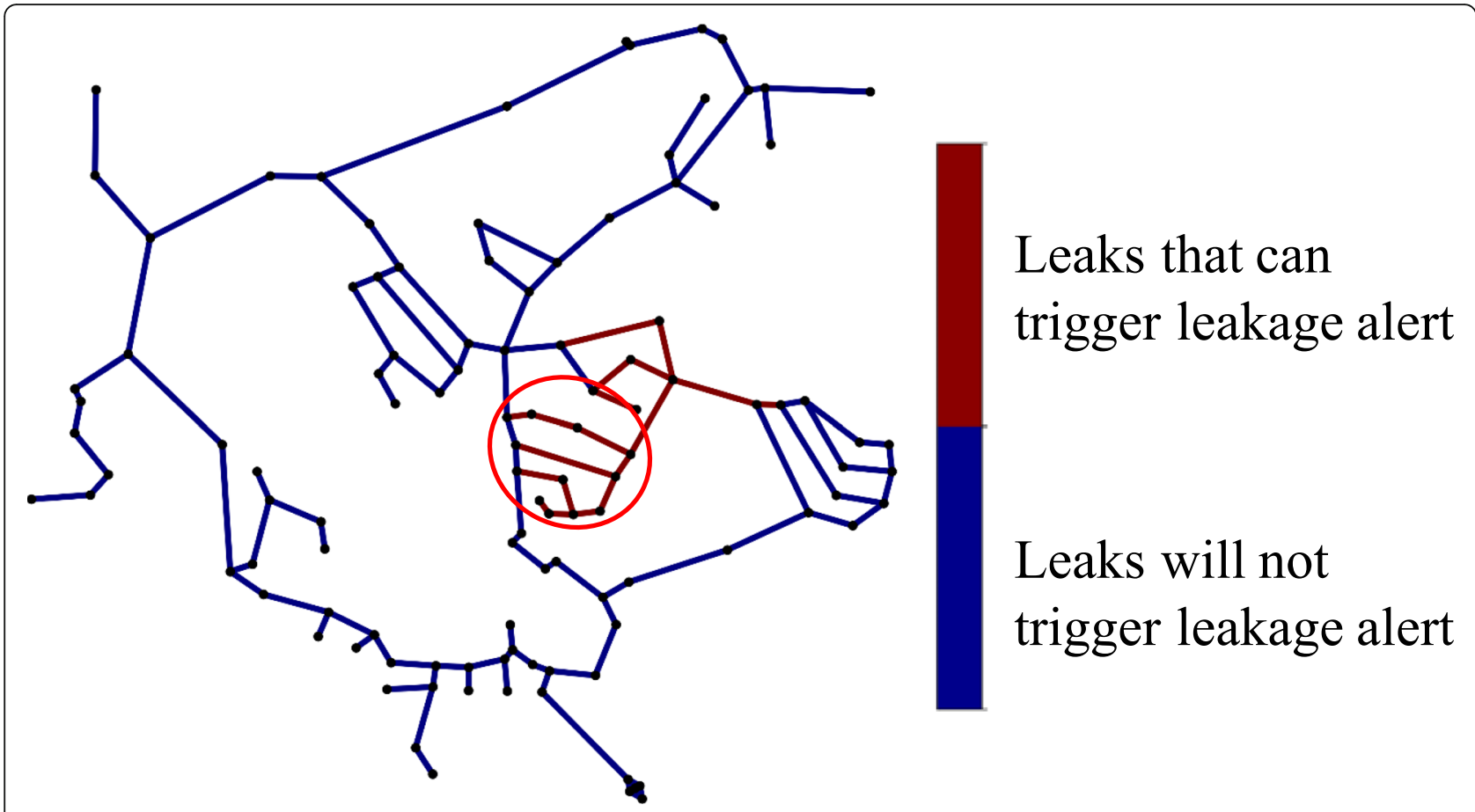

Fig. 16 Final leak detection result for each pipe leaking situation

area, the model can differential it from inside leaking situation to mitigate the false alert. There are two pipe leaking situations inside the monitoring area not detected. The main reason is the unappropriated threshold selection since all the pipes are using the same threshold. However, when more and more data available during the operation stage, this threshold can be tuned for each pipe, which will increase the detection ability eventually.

\section{Case study - II: C-town water distribution network}

C-Town water distribution network is a virtual network that was used for calibration competition in Battle of the Water Calibration Networks (BWCN) [33]. The topology and mode of operations of the network are described in details and the true network data are made public after the competition. This well-characterized water distribution network allows to test the proposed leaking detection method under a more complex scenario. From this, the performance of the proposed Autoencoder model based leak detection model is evaluated.

The topology of C-Town water distribution network is shown in Fig. 17. There are 1 reservoir and 7 water supply tanks. This network including 388 user nodes, 432 pipes, 11pumps, and 4 valves, which are divided into 5 district meter areas (DMA). The water demand at each node is provided. In this study, 4 predefined monitoring areas are chosen as shown in Fig. 17.
Hydraulic model of this C-Town WSN is built with WNTR. Since the water demand at each node is already defined, the actual water demands are used for hydraulic model rather than using the water demand ratio and uncertainty. The WDN under non-leaking situation and pipe failure (leaking size of $0.05 \mathrm{~m}$ ) are simulated. The leaking data set and non-leaking data set for the CTown WSN are generated following the same designed data generation procedures as described in Case study I Rancho Solano Zone III Water Distribution System.

Figure 18 shows the water pressure at node ' $\mathrm{J} 9$ ' with and without leaking. The leaking situation corresponding to leaking at pipe 'P251'. The exact position of node and pipe can also be found in Fig. 17.

\section{Performance of $A E$ model in leak detection}

The AE water pipe leakage detectors for each area is trained by using the samples from non-leaking data set. The performance of the AE leaking detection model is evaluated by calculating the probability of $\mathrm{AE}$ model triggering an alarm when leaking happens at each pipe, by sensors installed at different DMAs as shown in Fig. 17. For each pipe in the network, 169 sets of leaking and non-leaking water pressure data at nodes within the monitored area are generated. By feeding the water pressure data into the AE model, the probability successfully detection of each pipe leaking is shown in Fig. 19.

A few observations can be made from Fig. 19: 1) The probability of a successfully leak detection of a pipe is 

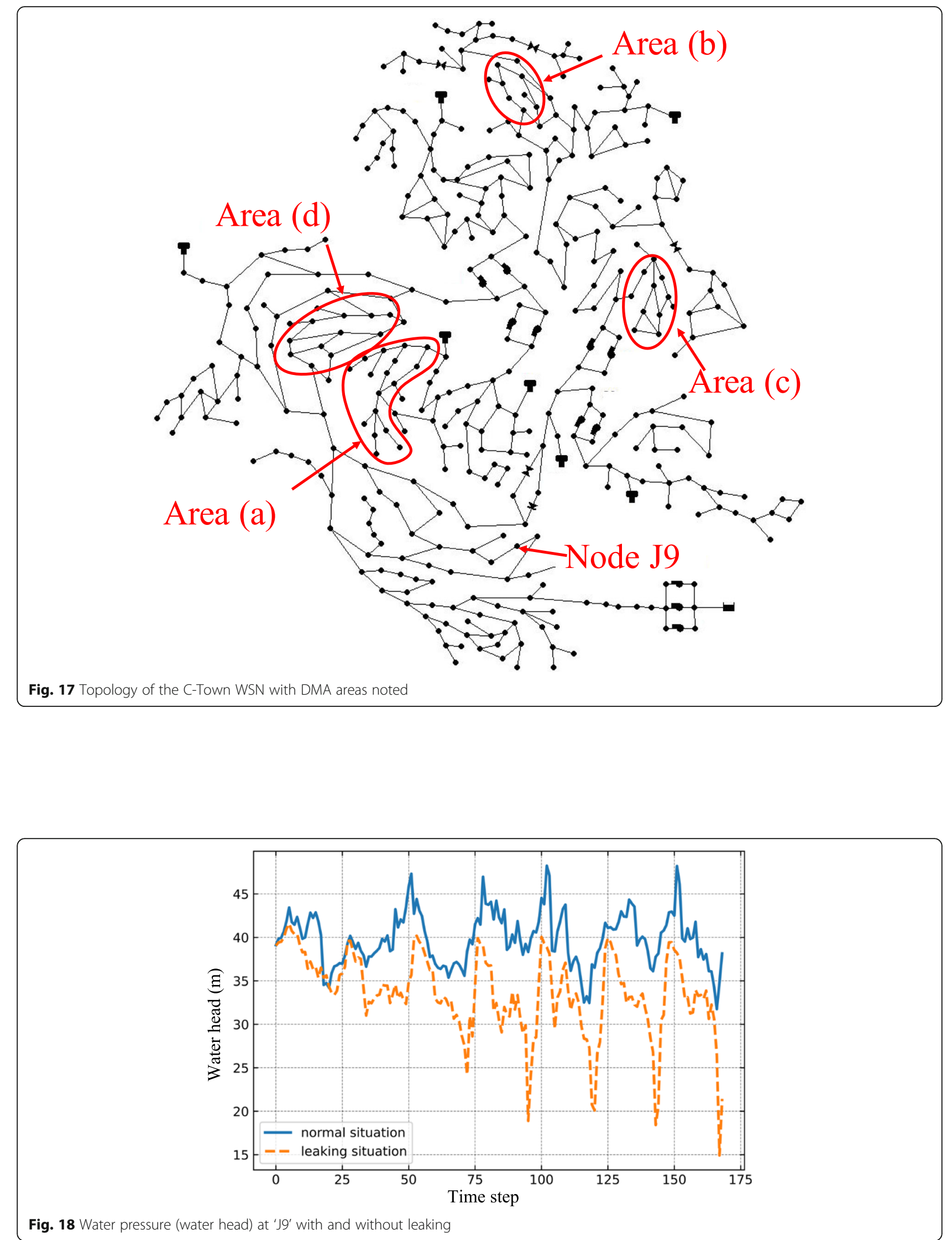


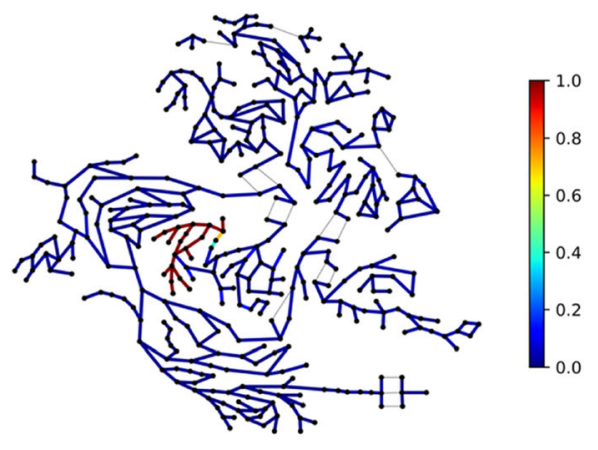

a)

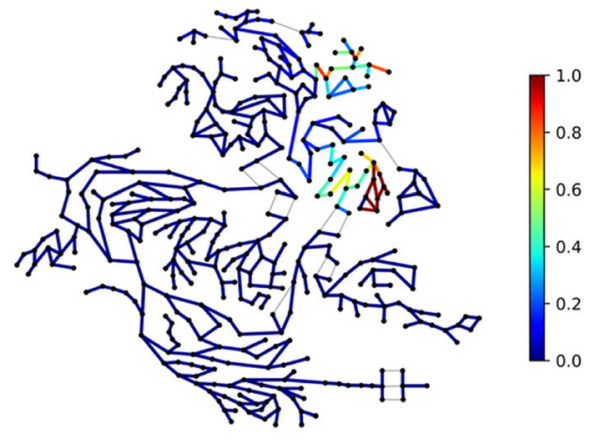

c)

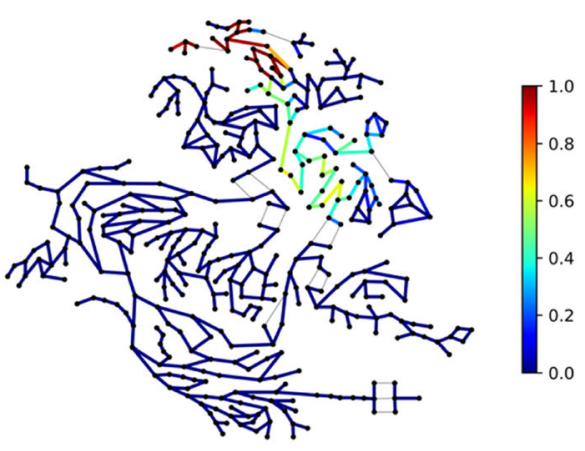

b)

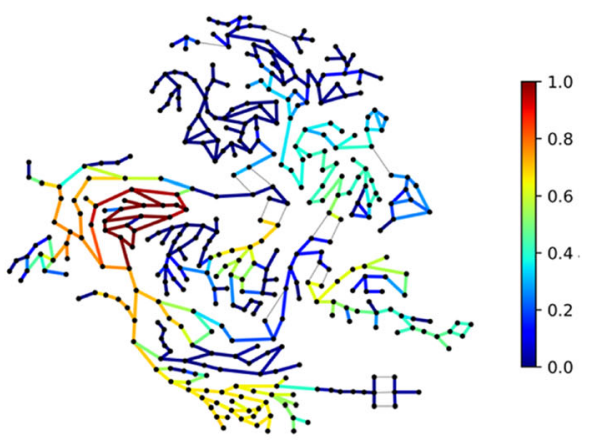

d)

Fig. 19 The probability of leak alert by AE detection model when leak happens on pipe in the WSN: a Monitoring sensors located in area a of Ctown, $\mathbf{c}$ Monitoring sensors located in area $\mathbf{b}$ of C-town, $\mathbf{c}$ Monitoring sensors located in area $\mathbf{c}$ of C-town, $\mathbf{d}$ Monitoring sensors located in area d of C-town

affected by the location of the pipe and the distribution of monitoring sensors. 2) the AE leak detection model achieved a high detection accuracy for leaks of pipe inside the monitoring area. 3) The probability of detecting leak in pipes outside the monitoring area is typically smaller and is affected by the topological structure of the WSN and setting of the AE model.

The proposed multiple detection attempts strategy is utilized to further improve the leaking detection accuracy and mitigate the false alarm. One hundred independent attempts are used. Leak alert will be triggered if more than 50 attempts indicated leaking (or reconstruction error by the $\mathrm{AE}$ model larger than the threshold). Fig. 20 shows the updated result of probability of detecting leaks in pipes in the WSN using this strategy. The results indicated leaks in pipes located in the monitoring area are all detected with $100 \%$ accuracy. Compared with the results shown in Fig. 18, the false alarm is significantly mitigated. In the meanwhile, leaks outside the monitoring area is not detected, except for Fig. 19 b). This is due to a conditional valve nearby and therefore leaks in these pipes have a larger disturbance to the WSN. The implication is that sensors need to be deployed in a strategic way to ensure the full coverage of the complete WSN.

\section{Conclusions and discussions}

Real time detection of leaks in the water supply network (WSN) bears important socio-economic benefits and is, however, challenging. Innovative data-driven machine learning (ML) models for leak detection are developed in this study. The spatial relationship of water pressure at multiple nodes in a water distribution network was learned and used for leak detection. Model-based data generation strategy is developed where data was generated by an industry certified hydraulic model for testbed WSNs. Factors such as the fluctuation of water demand are considered under both non-leaking and leaking situations. Artificial Neural Network (ANN) is found to achieve high accuracy in leak detection. It, however, requires balanced dataset including similar amount of data collected under leaking or non-leaking conditions, which 


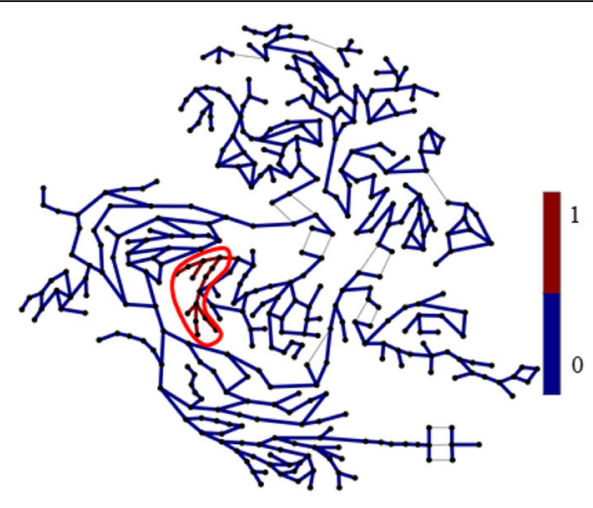

a)

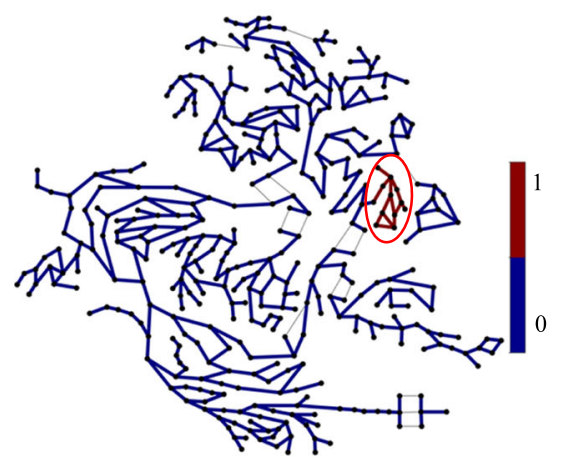

c)

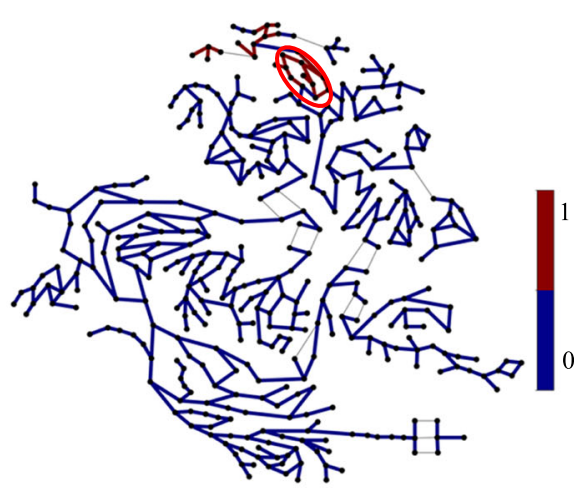

b)

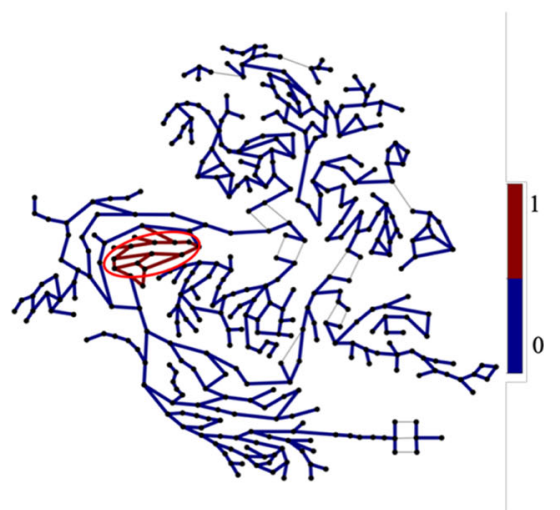

d)

Fig. 20 The probability of leak alert by AE detection model incorporating multiple attempts strategies for leak happens on pipe in the WSN: a Monitoring sensors located in DMA 4, c Monitoring sensors located located in DMA 2, c Monitoring sensors located in DMA 3, d Monitoring sensors located in DMA 1

is difficult to implement in the real WSNs where data under leaking condition is relative rare than that under normal conditions. The Autoencoder Neural Network (AE) model, an unsupervised ANN model, is developed to learn from the unbalanced data to classify the leaking versus non-leaking conditions. The results indicate $\mathrm{AE}$ achieved decent accuracy in leak detection. Factors affecting AE leak detection model are analyzed. An innovative strategy is proposed to further improve the reliability in leak detection by use of multiple independent attempts. The results show that this significantly reduces the false alarm. The AE based leak detection strategy is applied to a mature WSN to further evaluate its performance. The results indicated the $\mathrm{AE} \mathrm{ML}$ model-based strategy achieves high accuracy in detecting leaks in pipes located within the monitoring area. The accuracy in detecting leaks in a pipe is dependent upon the location of the water pipe and the distribution of monitoring sensor. The use of multiple attempts strategy significantly reduced the false alarm.

\section{Discussions}

Detecting leaks in the WSN is challenging due to the complex topology, fluctuations in user demand, and lack of monitoring data. Detecting leaks with inspection tools is expensive and labor-intensive, and cannot achieve real-time detection. Traditional model updating approach for structural health monitoring is difficult to implement for WSN due to complex topology and uncertainty in the hydraulic conditions. Detecting leaks based on the transient responses of WSN requires to capture the transient signals over a very short period when leak occurs, which requires high sampling rate. Data-driven approach using ML models is promising to achieve quick and reliable leak detection. The rationale is that the spatial pattern of water pressure and its variations under leak are affected by the network structure of water distribution offers information about the conditions of the WSN. The ML models developed in this study allow to detect leak from unbalanced data, i.e., with only data under 
normal operational conditions. Compared to other leak detection algorithms, the methods have the following advantages:

1) Provide quick leak detection with high accuracy.

2) Unlike the conventional transient-based leak detection, which requires sensor with high sampling rates to capture the transient process. The AE leak detection model learn from the spatial pattern contained in the data and only needs sensor with low sampling rate (and therefore inexpensive).

3) By using data from multiple nodes, the detection is more robust than data-driven models that only use data at single node.

4) The data driven approach does not require strong domain expertise to implement.

While data used for model training and validation in this study are from generated data by high fidelity model for WSN. The framework is readily applied to real world data. With the development of the Internet of Things (IoT), more and more sensors, i.e., smart meters, will be deployed into the water distribution network to monitor its health conditions. This will allow to obtain data to be used by the developed model.

Future work is recommended to continue to refine the ML model and evaluate its performance with the monitoring data from in-service WSNs. There are also many issues that require further study. For example, the ability of a monitoring sensor to monitor the change of water pressure caused by leakage may decrease with the increasing distance of the sensor to the leakage position. Therefore, how to optimize the distribution of monitoring sensor to effectively cover the whole WSN is an important issue. Strategies could include to divide a large WSN into several smaller districts, which could improve the coverage of the whole WSN in a cost effective way and provide reliable detection accuracy. Precisely locate the leaking position is important for timely intervention, which is also an important topic requiring further investigation.

\section{Abbreviations \\ WSN: Water supply network; ML: Machine Learning; ANN: Artificial neural network; AE: Autoencoder neural network; SCADA: Supervisory control and data acquisition; DMA: Districted metering area; SVM: Support Vector Machine; $N(u, \sigma)$ : Gaussian distribution with $u$ as mean and $\sigma$ as standard deviation; $U($,$) : Uniform distribution with lower bound and upper bound;$ MSE: Mean Square Error; loT: Internet of Things}

\section{Acknowledgements}

The author acknowledge the help from the staff members of the Cleveland Water Department led by Mr. Alex Margevicius during the study.

\section{Authors' contributions}

Xiong (Bill) Yu: envision the research, guide research activities; Xudong Fan: conduct analyses; Xijin Zhang: provide assistance. The authors read and approved the final manuscript.

\section{Funding}

This research is partially supported by the US National Science Foundation.

Availability of data and materials

The data and code are available upon request.

\section{Declarations}

Ethics approval and consent to participate

No human subject or animals are involved in the study.

\section{Consent for publication}

The authors consent the publications of this paper.

\section{Competing interests}

The authors declare that they have no competing interests.

\section{Author details}

${ }^{1}$ Department of Civil and Environmental Engineering, Case Western Reserve University, 2104 Adelbert Road, Bingham 248, Cleveland, OH 44106-7201, USA. ${ }^{2}$ Department of Civil and Environmental Engineering, Case Western Reserve University, 2104 Adelbert Road, Bingham 249C, Cleveland, OH 44106-7201, USA. '3 Department of Civil and Environmental Engineering, Case Western Reserve University, 2104 Adelbert Road, Bingham 206, Cleveland, OH 44106-7201, USA.

Received: 5 February 2021 Accepted: 16 March 2021

Published online: 15 April 2021

\section{References}

1. Abokifa AA, Haddad K et al (2018) Real-time identification of cyber-physical attacks on water distribution systems via machine learning-based anomaly detection techniques. J Water Resour Plan Manag 145(1):04018089

2. Adedeji KB, Hamam Y, Abe BT, Abu-Mahfouz AM (2017) Towards achieving a reliable leakage detection and localization algorithm for application in water piping networks: an overview. IEEE Access 5:20272-20285. https://doi. org/10.1109/ACCESS.2017.2752802

3. Amran TST, Ismail MP et al (2017) Detection of underground water distribution piping system and leakages using ground penetrating radar (GPR). AIP Conference Proceedings, AIP Publishing LLC

4. Bakker M, Vreeburg J et al (2013) A fully adaptive forecasting model for short-term drinking water demand. Environ Model Softw 48:141-151. https://doi.org/10.1016/j.envsoft.2013.06.012

5. Bimpas M, Amditis A, Uzunoglu N (2010) Detection of water leaks in supply pipes using continuous wave sensor operating at $2.45 \mathrm{GHz}$. J Appl Geophys 70(3):226-236. https://doi.org/10.1016/j.jappgeo.2010.01.003

6. Braun M, Piller O, Deuerlein J, Mortazavi I (2017) Limitations of demand-and pressure-driven modeling for large deficient networks. Drinking Water Eng Sci 10(2):93-98. https://doi.org/10.5194/dwes-10-93-2017

7. Buch N, Velastin SA, Orwell J (2011) A review of computer vision techniques for the analysis of urban traffic. IEEE Trans Intell Transp Syst 12(3):920-939. https://doi.org/10.1109/TITS.2011.2119372

8. Butler D (2000) Leakage detection and management: a comprehensive guide to technology and practice in the water supply industry. Palmer Environmental

9. Caputo AC, Pelagagge PM (2003) Using neural networks to monitor piping systems. Process Saf Prog 22(2):119-127. https://doi.org/10.1002/ prs.680220208

10. Chan TK, Chin CS, Zhong X (2018) Review of current technologies and proposed intelligent methodologies for water distributed network leakage detection. IEEE Access 6:78846-78867. https://doi.org/10.1109/ACCESS.201 8.2885444

11. Chim TW, Yiu S-M, Hui LCK, Li VOK (2011) SPECS: secure and privacy enhancing communications schemes for VANETs. Ad Hoc Netw 9(2):189203. https://doi.org/10.1016/j.adhoc.2010.05.005

12. Colombo AF, Lee P, Karney BW (2009) A selective literature review of transient-based leak detection methods. J Hydro Environ Res 2(4):212-227. https://doi.org/10.1016/j.jher.2009.02.003

13. Crowl DA, Louvar JF (2001) Chemical process safety: fundamentals with applications. Pearson Education 
14. De Coster A, Medina JP et al (2019) Towards an improvement of GPR-based detection of pipes and leaks in water distribution networks. J Appl Geophys 162:138-151. https://doi.org/10.1016/j.jappgeo.2019.02.001

15. Funk A, De Oreo WB (2011) Embedded energy in water studies study 3: end-use water demand profiles. Prepared by Aquacraft, Inc. for the California Public Utilities Commission Energy Division, Managed by California Institute for Energy and Environment, CALMAC Study ID CPU0052

16. Gao J, Shi B, Zhang W, Zhu H (2006) Monitoring the stress of the posttensioning cable using fiber optic distributed strain sensor. Measurement 39(5):420-428. https://doi.org/10.1016/j.measurement.2005.12.002

17. Hernadez E, Hoagland S et al (2016) Water distribution database for research applications. World Environ Water Resour Cong; 2016. p. 10. https://doi.org/10.1061/9780784479865.049.

18. Housh M, Ohar Z (2018) Model-based approach for cyber-physical attack detection in water distribution systems. Water Res 139:132-143. https://doi. org/10.1016/j.watres.2018.03.039

19. Jain AK, Mao J et al (1996) Artificial neural networks: a tutorial. Computer 3: $31-44$

20. Kang J, Park Y-J et al (2017) Novel leakage detection by ensemble CNN-SVM and graph-based localization in water distribution systems. IEEE Trans Ind Electron 65(5):4279-4289

21. Kiliç R (2016) Effective Management of Leakage in drinking water network. Acta Phys Pol A 130(1):479-483. https://doi.org/10.12693/APhysPolA.130.479

22. Kim J-H, Sharma G et al (2010) SPAMMS: a sensor-based pipeline autonomous monitoring and maintenance system. 2010 second international conference on COMmunication systems and NETworks (COMSNETS 2010), IEEE

23. Klise KA, Murray R et al (2018) An overview of the water network tool for resilience (WNTR). Sandia National lab. (SNL-NM), Albuquerque

24. Liao S-H, Chu P-H, Hsiao PY (2012) Data mining techniques and applications-a decade review from 2000 to 2011. Expert Syst Appl 39(12): 11303-11311. https://doi.org/10.1016/j.eswa.2012.02.063

25. Lin W-Y, Hu Y-H et al (2011) Machine learning in financial crisis prediction: a survey. IEEE Trans Syst Man Cybern Part C Appl Rev 42(4):421-436

26. Liou CP (1998) Limitations and proper use of the Hazen-Williams equation. J Hydraul Eng 124(9):951-954. https://doi.org/10.1061/(ASCE)0733-9429(1 998) $124: 9(951)$

27. Loth JL, Morris GJ et al (2004) Acoustic detecting and locating gas pipe line infringement. West Virginia University (US)

28. Mashford J, De Silva D et al (2012) Leak detection in simulated water pipe networks using SVM. Appl Artif Intell 26(5):429-444. https://doi.org/10.1080/ 08839514.2012 .670974

29. Mazzolani G, Berardi L, Laucelli D, Simone A, Martino R, Giustolisi O (2017) Estimating leakages in water distribution networks based only on inlet flow data. J Water Resour Plan Manag 143(6):04017014. https://doi.org/10.1061/ (ASCE)WR.1943-5452.0000758

30. Mounce S, Boxall J et al (2010a) Development and verification of an online artificial intelligence system for detection of bursts and other abnormal flows. J Water Resour Plan Manag 136(3):309-318. https://doi.org/10.1061/ (ASCE)WR.1943-5452.0000030

31. Mounce SR, Day AJ, Wood AS, Khan A, Widdop PD, Machell J (2002) A neural network approach to burst detection. Water Sci Technol 45(4-5):237246. https://doi.org/10.2166/wst.2002.0595

32. Mounce SR, Mounce RB et al (2010b) Novelty detection for time series data analysis in water distribution systems using support vector machines. J Hydroinf 13(4):672-686

33. Ostfeld A, Salomons E, Ormsbee L, Uber JG, Bros CM, Kalungi P, Burd R, Zazula-Coetzee B, Belrain T, Kang D, Lansey K, Shen H, McBean E, Yi Wu Z, Walski T, Alvisi S, Franchini M, Johnson JP, Ghimire SR, Barkdoll BD, Koppel T, Vassiljev A, Kim JH, Chung G, Yoo DG, Diao K, Zhou Y, Li J, Liu Z, Chang K, Gao J, Qu S, Yuan Y, Prasad TD, Laucelli D, Vamvakeridou Lyroudia LS, Kapelan Z, Savic D, Berardi L, Barbaro G, Giustolisi O, Asadzadeh M, Tolson BA, McKillop R (2012) Battle of the water calibration networks. J Water Resour Plan Manag 138(5):523-532. https://doi.org/10.1061/(ASCE)WR.19435452.0000191

34. Ozevin D, Yalcinkaya H (2012) Reliable monitoring of leak in gas pipelines using acoustic emission method. Proc. Civil Struct. Health Monit. Workshop (CSHM)

35. Pal A, Kant K (2019) Water flow driven sensor networks for leakage and contamination monitoring in distribution pipelines. ACM Transact Sensor Netw (TOSN) 15(4):1-43. https://doi.org/10.1145/3342513
36. Prasad TD, Park N-SJJOWRP et al (2004) Multiobjective genetic algorithms for design of water distribution networks. J. Water Resour Plan Manag. 130(1):73-82

37. Romano M, Kapelan Z et al (2012) Automated detection of pipe bursts and other events in water distribution systems. J Water Resour Plan Manag 140(4):457-467

38. Sadeghioon A, Metje N, Chapman D, Anthony C (2014) SmartPipes: smart wireless sensor networks for leak detection in water pipelines. J Sens Actuator Netw 3(1):64-78. https://doi.org/10.3390/jsan3010064

39. Sadiq R, Rajani B, Kleiner Y (2004) Probabilistic risk analysis of corrosion associated failures in cast iron water mains. Reliab Eng Syst Saf 86(1):1-10. https://doi.org/10.1016/j.ress.2003.12.007

40. Shanker M, Hu MY, Hung MS (1996) Effect of data standardization on neural network training. Omega 24(4):385-397. https://doi.org/10.1016/0305-0483 (96)00010-2

41. Srirangarajan S, Allen M, Preis A, Iqbal M, Lim HB, Whittle AJ (2013) Waveletbased burst event detection and localization in water distribution systems. J Sign Proc Syst 72(1):1-16. https://doi.org/10.1007/s11265-012-0690-6

42. Srirangarajan S, lqbal M et al (2010) Water main burst event detection and localization. Water Distrib Syst Anal 2010:1324-1335

43. Stoianov I, Nachman L et al (2007) PIPENETa wireless sensor network for pipeline monitoring. Proceedings of the 6th international conference on information processing in sensor networks

44. Tao T, Huang $\mathrm{H}$ et al (2013) Burst detection using an artificial immune network in water-distribution systems. J Water Resour Plan Manag 140(10): 04014027

45. Taormina R, Galelli S, Tippenhauer NO, Salomons E, Ostfeld A, Eliades DG, Aghashahi M, Sundararajan R, Pourahmadi M, Banks MK, Brentan BM, Campbell E, Lima G, Manzi D, Ayala-Cabrera D, Herrera M, Montalvo I, Izquierdo J, Luvizotto E Jr, Chandy SE, Rasekh A, Barker ZA, Campbell B, Shafiee ME, Giacomoni M, Gatsis N, Taha A, Abokifa AA, Haddad K, Lo CS, Biswas P, Pasha MFK, Kc B, Somasundaram SL, Housh M, Ohar Z (2018) Battle of the attack detection algorithms: disclosing cyber attacks on water distribution networks. J Water Resour Plan Manag 144(8):04018048. https:// doi.org/10.1061/(ASCE)WR.1943-5452.0000969

46. Twort AC, Ratnayaka DD et al (2000) Water supply. Elsevier

47. Walski TM, Male JW (2000) Maintenance and rehabilitation/replacement. McGraw-Hill 17:11-17.28

48. Wu Y, Liu S (2017) A review of data-driven approaches for burst detection in water distribution systems. Urban Water J 14(9):972-983. https://doi.org/1 $0.1080 / 1573062 X .2017 .1279191$

49. Ye G, Fenner RA (2011) Kalman filtering of hydraulic measurements for burst detection in water distribution systems. J Pipeline Syst Eng Pract 2(1):14-22. https://doi.org/10.1061/(ASCE)PS.1949-1204.0000070

50. Zhou X, Tang Z, Xu W, Meng F, Chu X, Xin K, Fu G (2019) Deep learning identifies accurate burst locations in water distribution networks. Water Res 166:115058. https://doi.org/10.1016/j.watres.2019.115058

\section{Publisher's Note}

Springer Nature remains neutral with regard to jurisdictional claims in published maps and institutional affiliations.

\section{Submit your manuscript to a SpringerOpen ${ }^{\circ}$ journal and benefit from:}

- Convenient online submission

- Rigorous peer review

- Open access: articles freely available online

- High visibility within the field

- Retaining the copyright to your article

Submit your next manuscript at $>$ springeropen.com 